\title{
Angewandte
}

Supporting Information

(c) Wiley-VCH 2006

69451 Weinheim, Germany 


\section{Towards a General Scale of Nucleophilicity?}

Thanh Binh Phan, Martin Breugst, and Herbert Mayr*

* Department Chemie und Biochemie der Ludwig-Maximilians-Universität München,

Butenandtstrasse 5-13 (Haus F), 81377 München, Germany.

Telephone: (+49) 89-2180-77719. Fax: (+49) 89-2180-77717.

E-mail: Herbert.Mayr@cup.uni-muenchen.de

\section{CONTENTS}

1. Chemicals S2

2. Determination of nucleophilicity parameters in methanol S2

2.1. Trimethyl phosphite S4

2.2. Trifluoroethylamine S5

2.3. Imidazole $\quad$ S6

$\begin{array}{ll}\text { 2.4. Hydroxylamine } & \text { S7 }\end{array}$

2.5. Ethanolamine $\quad$ S8

2.6. $n$-Propylamine $\quad$ S9

2.7. Benzylamine $\quad \mathrm{S} 10$

2.8. Hydrazine $\quad \mathrm{S} 11$

2.9. Diethanolamine $\quad \mathrm{S} 12$

2.10. Morpholine S13

$\begin{array}{lr}\text { 2.11. Piperidine } & \text { S14 }\end{array}$

2.12. Pyrrolidine S15

3. Rate constants of the reactions of the S-methyldibenzothiophenium ion with nucleophiles $\quad \mathbf{S 1 7}$

$\begin{array}{ll}\text { 3.1. Kinetics of the reactions with different nucleophiles } & \text { S18 }\end{array}$

3.2. Kinetics of the reactions with hydroxylamine and hydrazine $\quad$ S20

$\begin{array}{ll}\text { 3.3. Kinetics of the reactions with carbanions } & \text { S21 }\end{array}$

3.4. Kinetics of the reactions with propan-1-ol and propan-2-ol S23

References $\quad$ S24 


\section{Chemicals}

Solvents. Reagent grade methanol ( $>99.8 \%$, Acros), anhydrous propan-2-ol ( $>99.5 \%$, Aldrich), and anhydrous acetonitrile ( $>99.5 \%$, Merck) were purchased. Propan-1-ol was distilled from magnesium and iodine.

Electrophiles. Benzhydrylium tetrafluoroborates were prepared as described in reference [S1], $S$ methyldibenzothiophenium trifluoromethanesulfonate was prepared according to the procedure described by Kevill and Anderson. ${ }^{[\mathrm{S} 2]}$

Nucleophiles. Trimethyl phosphite ( $>99.99 \%$, Aldrich), 2,2,2-trifluoroethylamine ( $>99.5 \%$, Aldrich), imidazole ( $>99.5 \%$, Fluka), $n$-propylamine ( $>99.5 \%$, Fluka), benzylamine ( $>99.5 \%$, Fluka), hydroxylamine hydrochloride ( $>99 \%$, Fluka), hydrazine dihydrochloride ( $>99 \%$, Fluka), ethanolamine ( $>99 \%$, Acros), diethanolamine ( $>99 \%$, Acros), sodium methoxide ( $>99.8 \%$, Acros), and sodium azide ( $>99 \%$, Merck) were purchased and used without further purification. Piperidine ( $>99 \%$, Lancaster), pyrrolidine ( $>99 \%$, Lancaster), morpholine ( $>99 \%$, Lancaster), malononitrile ( $>99 \%$, Lancaster), and dimethyl malonate ( $>99$ $\%$, Lancaster) were purchased and freshly distilled before use.

\section{Determination of nucleophilicity parameters in methanol}

The reactivity parameters $N$ and $s_{\mathrm{N}}$ of various nucleophiles in methanol were determined on the basis of Equation (S1) using benzhydrylium ions (Table S1) as reference electrophiles. ${ }^{[\mathrm{S} 1]}$

$$
\log k\left(20^{\circ} \mathrm{C}\right)=s_{\mathrm{N}}(N+E)
$$

Since the reactions of the colored reference electrophiles with nucleophiles gave rise to colorless products, the decay of the absorptions of the electrophiles was followed by UV-Vis spectroscopy. ${ }^{[\mathrm{S} 3]}$

Because of the fast reactions of most benzhydrylium ions with methanol, it was necessary to prepare stock solutions of benzhydrylium salts in acetonitrile. Therefore, the reactions were followed in methanol with a content of $9 \%(\mathrm{v} / \mathrm{v})$ of acetonitrile. Such solutions were obtained by mixing 10 parts of a solution of nucleophiles in methanol with 1 part of a solution of a benzhydrylium tetrafluoroborate in acetonitrile in a stopped-flow instrument. Hi-Tech SF-61DX2 stopped-flow spectrophotometer systems (controlled by HiTech KinetAsyst 2 software) were used for the investigation of the reactions. The temperature of the solutions during all kinetic studies was kept constant $\left(20.0 \pm 0.1^{\circ} \mathrm{C}\right)$ by using a circulating bath thermostat.

The concentrations of the nucleophiles $(\mathrm{Nu})$ were at least ten times as high as those of the electrophiles, resulting in first-order kinetics with an exponential decay of the electrophile concentrations. The first-order rate constants $k_{\mathrm{obs}}\left(\mathrm{s}^{-1}\right)$ were obtained by least-squares fitting of the absorbance $A$ of the electrophiles to the single-exponential $A_{\mathrm{t}}=A_{\mathrm{o}} \exp \left(-k_{\mathrm{obs}} t\right)+C$. Because of $k_{\mathrm{obs}}=k_{2}[\mathrm{Nu}]$, second-order rate constants $k_{2}\left(\mathrm{M}^{-1} \mathrm{~s}^{-1}\right)$ were derived from the slopes of the linear plots of $k_{\mathrm{obs}}\left(\mathrm{s}^{-1}\right) \mathrm{vs}$. [Nu].

The linear plots of $\log k_{2}$ vs. the electrophilicity parameters $E$ (Table S1) of the benzhydrylium ions yield the nucleophilicity parameters $N$ and $s$ of the nucleophiles in methanol according to eq. S1. 
Table S1. Benzhydrylium ions $\left(\mathrm{Ar}_{2} \mathrm{CH}^{+}\right)$used as reference electrophiles in this work.

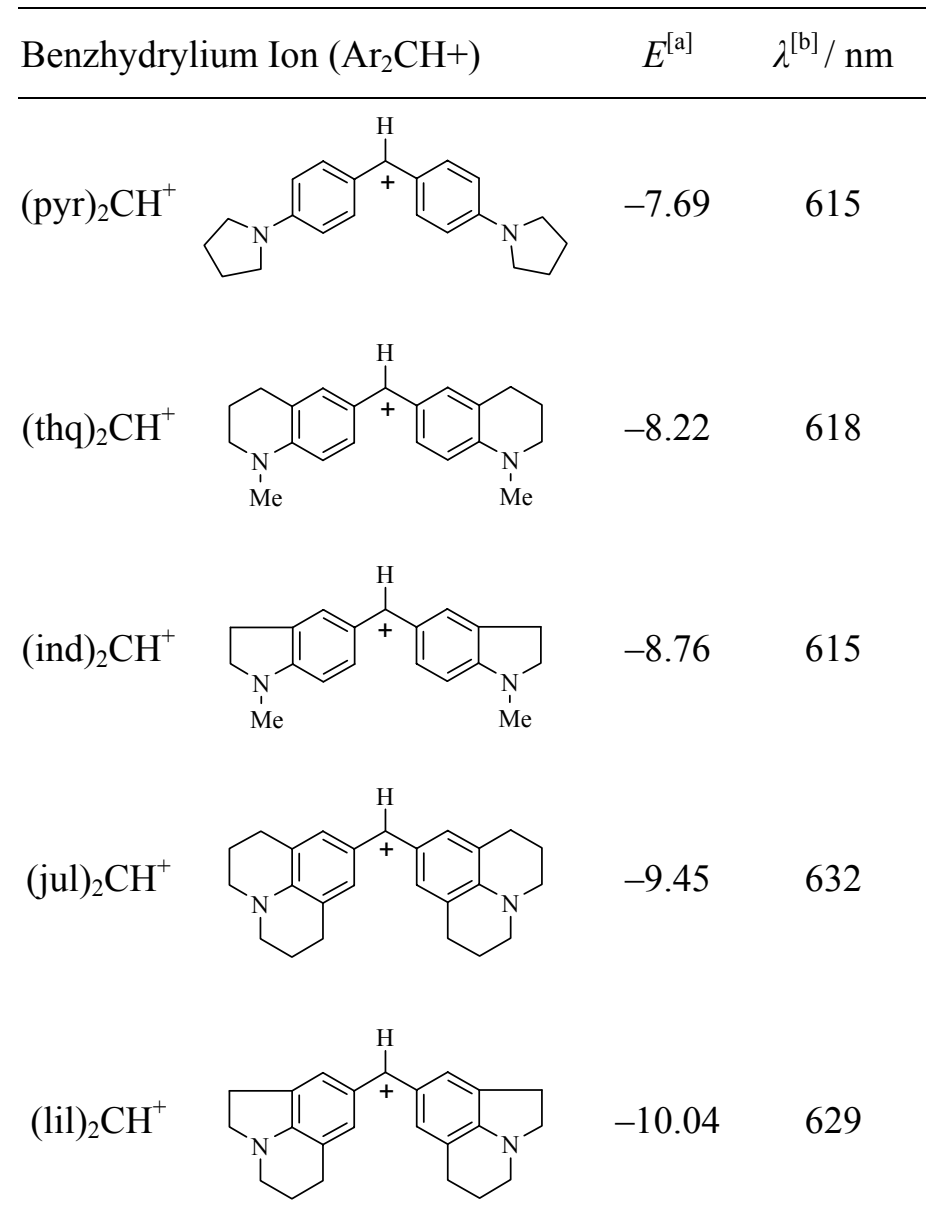

[a] From reference [S1]; [b] Wavelenghts at which the reactions were followed.

For the reasons discussed above, the kinetic data reported in this part have not been determined in pure methanol, but in $91 / 9(\mathrm{v} / \mathrm{v})$ methanol/acetonitrile mixtures. However, this content of acetonitrile has been proven to have only little influence on the rate constants of the reactions of nucleophiles with benzhydrylium ions. ${ }^{[\mathrm{S} 4]}$ Therefore, one can consider the rate constants obtained in $91 / 9(\mathrm{v} / \mathrm{v})$ methanol/acetonitrile mixtures to closely resemble those for reactions in pure methanol. 


\subsection{Trimethyl phosphite}

Kinetics of the reactions with benzhydrylium ions $\left(20^{\circ} \mathrm{C}\right.$, in $91 / 9$ (v/v) $\mathrm{MeOH} / \mathrm{MeCN}$; stopped-flow)

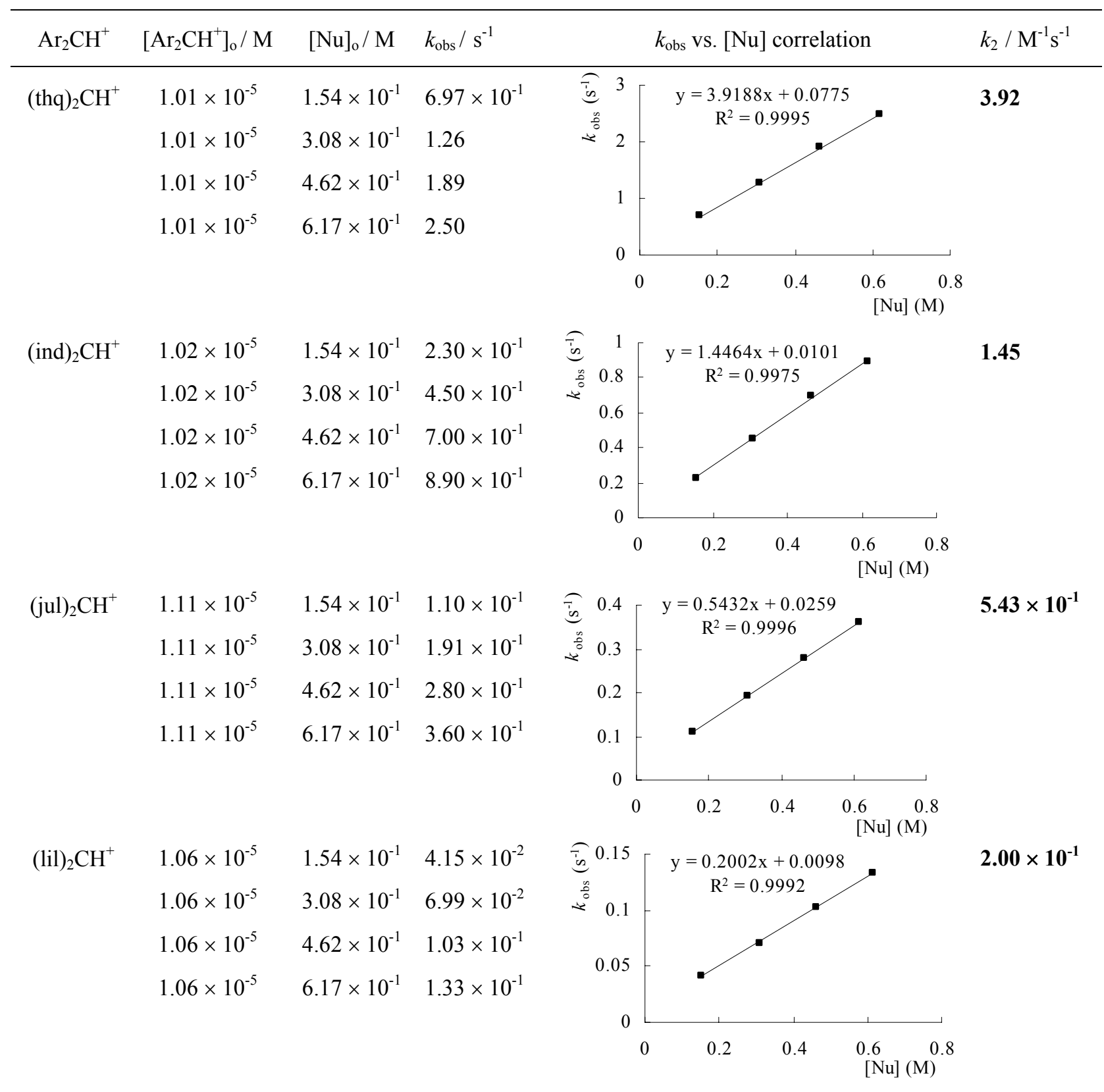

Reactivity parameters: $N=9.04 ; s_{\mathrm{N}}=0.698$

\begin{tabular}{llll}
\hline Electrophile & \multicolumn{1}{c}{$E$} & \multicolumn{1}{c}{$k_{2} / \mathrm{M}^{-1} \mathrm{~s}^{-1}$} & $\log k_{2}$ \\
\hline (thq $)_{2} \mathrm{CH}^{+}$ & -8.22 & 3.92 & 0.59 \\
(ind $)_{2} \mathrm{CH}^{+}$ & -8.76 & 1.45 & 0.16 \\
(jul) $\mathrm{CH}^{+}$ & -9.45 & $5.43 \times 10^{-1}$ & -0.27 \\
$(\text { lil })_{2} \mathrm{CH}^{+}$ & -10.04 & $2.00 \times 10^{-1}$ & -0.70 \\
\hline
\end{tabular}

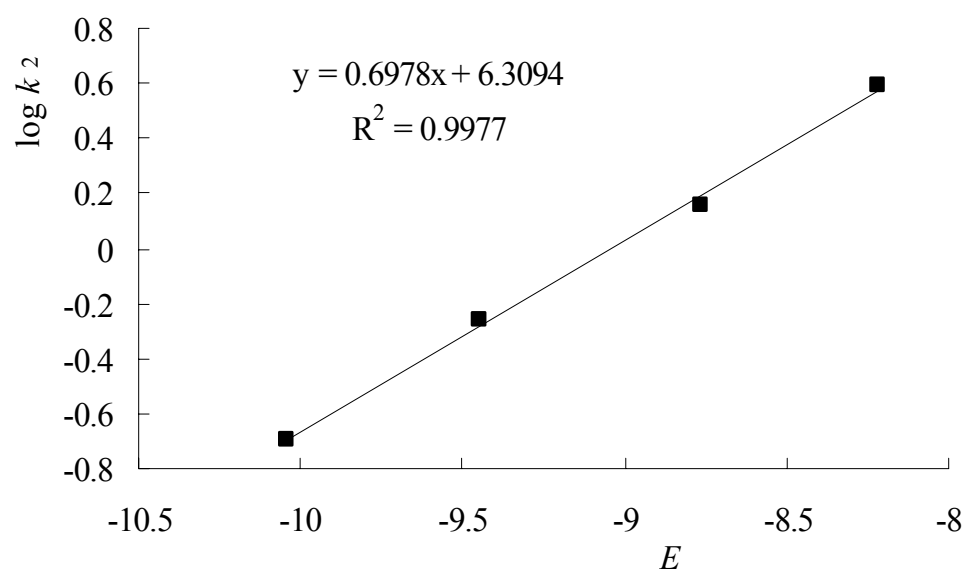




\subsection{2,2,2-Trifluoroethylamine}

Kinetics of the reactions with benzhydrylium ions $\left(20^{\circ} \mathrm{C}\right.$, in $91 / 9$ (v/v) $\mathrm{MeOH} / \mathrm{MeCN}$; stopped-flow)

\begin{tabular}{|c|c|c|c|c|c|c|}
\hline $\mathrm{Ar}_{2} \mathrm{CH}^{+}$ & {$\left[\mathrm{Ar}_{2} \mathrm{CH}^{+}\right]_{\mathrm{o}} / \mathrm{M}$} & {$[\mathrm{Nu}]_{\mathrm{o}} / \mathrm{M}$} & $k_{\mathrm{obs}} / \mathrm{s}^{-1}$ & & $k_{\text {obs }}$ vs. $[\mathrm{Nu}]$ correlation & $k_{2} / \mathrm{M}^{-1} \mathrm{~s}^{-1}$ \\
\hline \multirow[t]{5}{*}{ (thq) $)_{2} \mathrm{CH}^{+}$} & $1.02 \times 10^{-5}$ & $2.71 \times 10^{-3}$ & $2.86 \times 10^{-1}$ & \multirow{2}{*}{$\overbrace{\substack{\infty \\
\infty}}^{\infty} 0.8$} & \multirow{2}{*}{$\begin{array}{c}y=66.996 x+0.1109 \\
R^{2}=0.9991\end{array}$} & \multirow[t]{5}{*}{$6.70 \times 10^{1}$} \\
\hline & $1.02 \times 10^{-5}$ & $5.41 \times 10^{-3}$ & $4.80 \times 10^{-1}$ & & & \\
\hline & $1.02 \times 10^{-5}$ & $8.12 \times 10^{-3}$ & $6.60 \times 10^{-1}$ & 0.4 & & \\
\hline & $1.02 \times 10^{-5}$ & $1.08 \times 10^{-2}$ & $8.30 \times 10^{-1}$ & 0.2 & & \\
\hline & & & & & $\begin{array}{ccc}0.005 & 0.01 & 0.015 \\
& {[\mathrm{Nu}](\mathrm{M})}\end{array}$ & \\
\hline \multirow[t]{5}{*}{ (ind) ${ }_{2} \mathrm{CH}^{+}$} & $1.05 \times 10^{-5}$ & $2.71 \times 10^{-3}$ & $1.30 \times 10^{-1}$ & \multirow{3}{*}{ 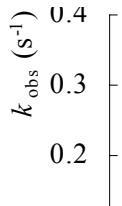 } & $\begin{array}{c}\mathrm{y}=19.946 \mathrm{x}+0.0752 \\
\mathrm{R}^{2}=0.9986\end{array}$ & \multirow[t]{3}{*}{$1.99 \times 10^{1}$} \\
\hline & $1.05 \times 10^{-5}$ & $5.41 \times 10^{-3}$ & $1.80 \times 10^{-1}$ & & & \\
\hline & $1.05 \times 10^{-5}$ & $8.12 \times 10^{-3}$ & $2.40 \times 10^{-1}$ & & & \\
\hline & $1.05 \times 10^{-5}$ & $1.08 \times 10^{-2}$ & $2.90 \times 10^{-1}$ & 0.1 & & \\
\hline & & & & & $\begin{array}{l}0.01 \quad 0.015 \\
{[\mathrm{Nu}](\mathrm{M})}\end{array}$ & \\
\hline \multirow[t]{5}{*}{$(\mathrm{jul})_{2} \mathrm{CH}^{+}$} & $1.01 \times 10^{-5}$ & $6.68 \times 10^{-3}$ & $9.30 \times 10^{-2}$ & \multirow{4}{*}{$\underbrace{0.2}_{0.1}$} & \multirow{4}{*}{$\begin{array}{c}\mathrm{y}=4.9574 \mathrm{x}+0.0613 \\
\mathrm{R}^{2}=0.9978\end{array}$} & \multirow[t]{5}{*}{4.96} \\
\hline & $1.01 \times 10^{-5}$ & $1.34 \times 10^{-2}$ & $1.30 \times 10^{-1}$ & & & \\
\hline & $1.01 \times 10^{-5}$ & $2.00 \times 10^{-2}$ & $1.60 \times 10^{-1}$ & & & \\
\hline & $1.01 \times 10^{-5}$ & $2.40 \times 10^{-2}$ & $1.80 \times 10^{-1}$ & & & \\
\hline & & & & 0 & 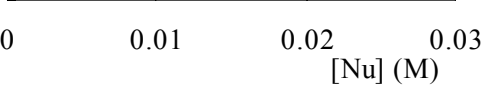 & \\
\hline
\end{tabular}

Reactivity parameters: $N=10.20 ; s_{\mathrm{N}}=0.917$

\begin{tabular}{lccc}
\hline Electrophile & $E$ & $k_{2} / \mathrm{M}^{-1} \mathrm{~s}^{-1}$ & $\log k_{2}$ \\
\hline (thq) ${ }_{2} \mathrm{CH}^{+}$ & -8.22 & 67.0 & 1.83 \\
(ind) ${ }_{2} \mathrm{CH}^{+}$ & -8.76 & 19.9 & 1.30 \\
(jul) ${ }_{2} \mathrm{CH}^{+}$ & -9.45 & 4.96 & 0.70 \\
\hline
\end{tabular}

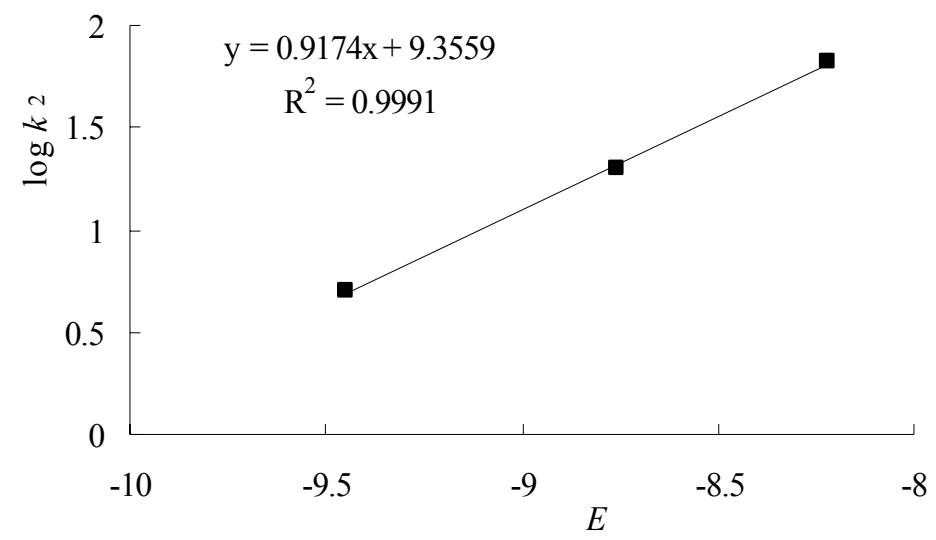




\subsection{Imidazole}

Kinetics of the reactions with benzhydrylium ions $\left(20^{\circ} \mathrm{C}\right.$, in $91 / 9$ (v/v) $\mathrm{MeOH} / \mathrm{MeCN}$; stopped-flow)

\begin{tabular}{|c|c|c|c|c|c|c|}
\hline $\mathrm{Ar}_{2} \mathrm{CH}^{+}$ & {$\left[\mathrm{Ar}_{2} \mathrm{CH}^{+}\right]_{\mathrm{o}} / \mathrm{M}$} & {$[\mathrm{Nu}]_{\mathrm{o}} / \mathrm{M}$} & $k_{\mathrm{obs}} / \mathrm{s}^{-1}$ & & $k_{\text {obs }}$ vs. $[\mathrm{Nu}]$ correlation & $k_{2} / \mathrm{M}^{-1} \mathrm{~s}^{-1}$ \\
\hline \multirow[t]{5}{*}{ (thq) ${ }_{2} \mathrm{CH}^{+}$} & $1.02 \times 10^{-5}$ & $6.80 \times 10^{-2}$ & 2.70 & \multirow{3}{*}{ 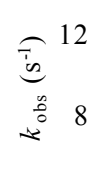 } & $y=34.944 x+0.3295$ & \multirow[t]{5}{*}{$3.49 \times 10^{1}$} \\
\hline & $1.02 \times 10^{-5}$ & $1.36 \times 10^{-1}$ & 5.10 & & $\mathrm{R}^{2}=1$ & \\
\hline & $1.02 \times 10^{-5}$ & $2.04 \times 10^{-1}$ & 7.43 & & & \\
\hline & $1.02 \times 10^{-5}$ & $2.72 \times 10^{-1}$ & 9.85 & 4 & & \\
\hline & & & & & $0.2_{[\mathrm{Nu}](\mathrm{M})}^{0.3}$ & \\
\hline \multirow[t]{5}{*}{ (ind) ${ }_{2} \mathrm{CH}^{+}$} & $1.06 \times 10^{-5}$ & $6.80 \times 10^{-2}$ & 1.03 & \multirow{3}{*}{ 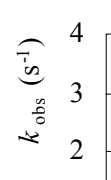 } & \multirow{4}{*}{$\begin{array}{c}y=13.139 x+0.136 \\
R^{2}=1\end{array}$} & \multirow[t]{5}{*}{$1.31 \times 10^{1}$} \\
\hline & $1.06 \times 10^{-5}$ & $1.36 \times 10^{-1}$ & 1.92 & & & \\
\hline & $1.06 \times 10^{-5}$ & $2.04 \times 10^{-1}$ & 2.82 & & & \\
\hline & $1.06 \times 10^{-5}$ & $2.72 \times 10^{-1}$ & 3.71 & 1 & & \\
\hline & & & & & $\begin{array}{l}0.2 \quad 0.3 \\
{[\mathrm{Nu}](\mathrm{M})}\end{array}$ & \\
\hline \multirow[t]{5}{*}{$(\mathrm{jul})_{2} \mathrm{CH}^{+}$} & $1.04 \times 10^{-5}$ & $6.80 \times 10^{-2}$ & $3.55 \times 10^{-1}$ & \multirow{4}{*}{ 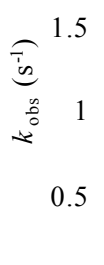 } & \multirow{4}{*}{$\begin{array}{c}\mathrm{y}=4.581 \mathrm{x}+0.0449 \\
\mathrm{R}^{2}=1\end{array}$} & \multirow[t]{5}{*}{4.58} \\
\hline & $1.04 \times 10^{-5}$ & $1.36 \times 10^{-1}$ & $6.70 \times 10^{-1}$ & & & \\
\hline & $1.04 \times 10^{-5}$ & $2.04 \times 10^{-1}$ & $9.80 \times 10^{-1}$ & & & \\
\hline & $1.04 \times 10^{-5}$ & $2.72 \times 10^{-1}$ & 1.29 & & & \\
\hline & & & & & $0.2 \quad 0.3$ & \\
\hline \multirow[t]{5}{*}{$(\text { lil })_{2} \mathrm{CH}^{+}$} & $1.03 \times 10^{-5}$ & $6.80 \times 10^{-2}$ & $1.53 \times 10^{-1}$ & \multirow{5}{*}{ 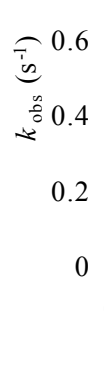 } & \multirow{4}{*}{$\begin{array}{c}\mathrm{y}=1.8452 \mathrm{x}+0.0281 \\
\mathrm{R}^{2}=1\end{array}$} & \multirow[t]{5}{*}{1.85} \\
\hline & $1.03 \times 10^{-5}$ & $1.36 \times 10^{-1}$ & $2.80 \times 10^{-1}$ & & & \\
\hline & $1.03 \times 10^{-5}$ & $2.04 \times 10^{-1}$ & $4.04 \times 10^{-1}$ & & & \\
\hline & $1.03 \times 10^{-5}$ & $2.72 \times 10^{-1}$ & $5.30 \times 10^{-1}$ & & & \\
\hline & & & & & $\begin{array}{l}0.2 \quad 0.3 \\
{[\mathrm{Nu}](\mathrm{M})}\end{array}$ & \\
\hline
\end{tabular}

Reactivity parameters: $N=10.41 ; s_{\mathrm{N}}=0.696$

\begin{tabular}{lccc}
\hline Electrophile & $E$ & $k_{2} / \mathrm{M}^{-1} \mathrm{~s}^{-1}$ & $\log k_{2}$ \\
\hline (thq $)_{2} \mathrm{CH}^{+}$ & -8.22 & 34.9 & 1.54 \\
(ind $)_{2} \mathrm{CH}^{+}$ & -8.76 & 13.1 & 1.12 \\
(jul $)_{2} \mathrm{CH}^{+}$ & -9.45 & 4.58 & 0.66 \\
$(\text { lil })_{2} \mathrm{CH}^{+}$ & -10.04 & 1.85 & 0.27 \\
\hline
\end{tabular}

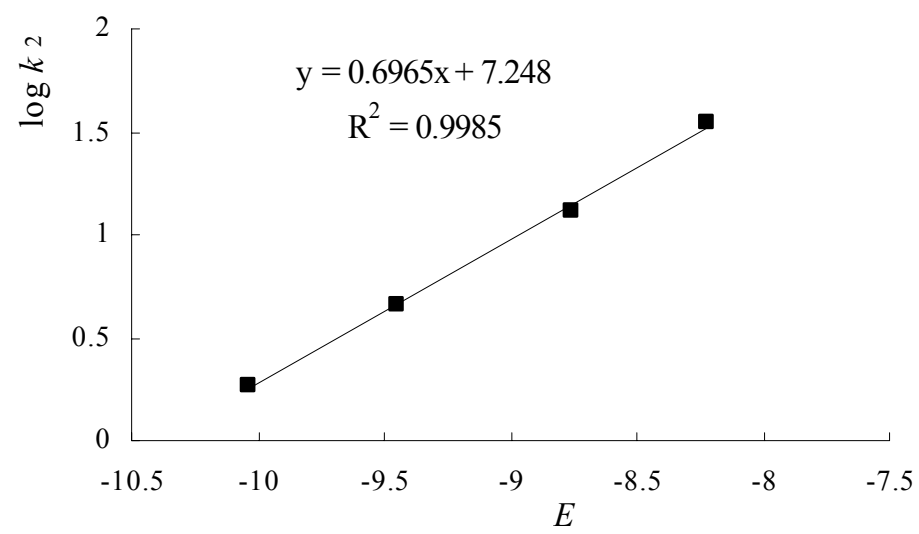




\subsection{Hydroxylamine}

Kinetics of the reactions with benzhydrylium ions $\left(20^{\circ} \mathrm{C}\right.$, in $91 / 9$ (v/v) $\mathrm{MeOH} / \mathrm{MeCN}$; stopped-flow)

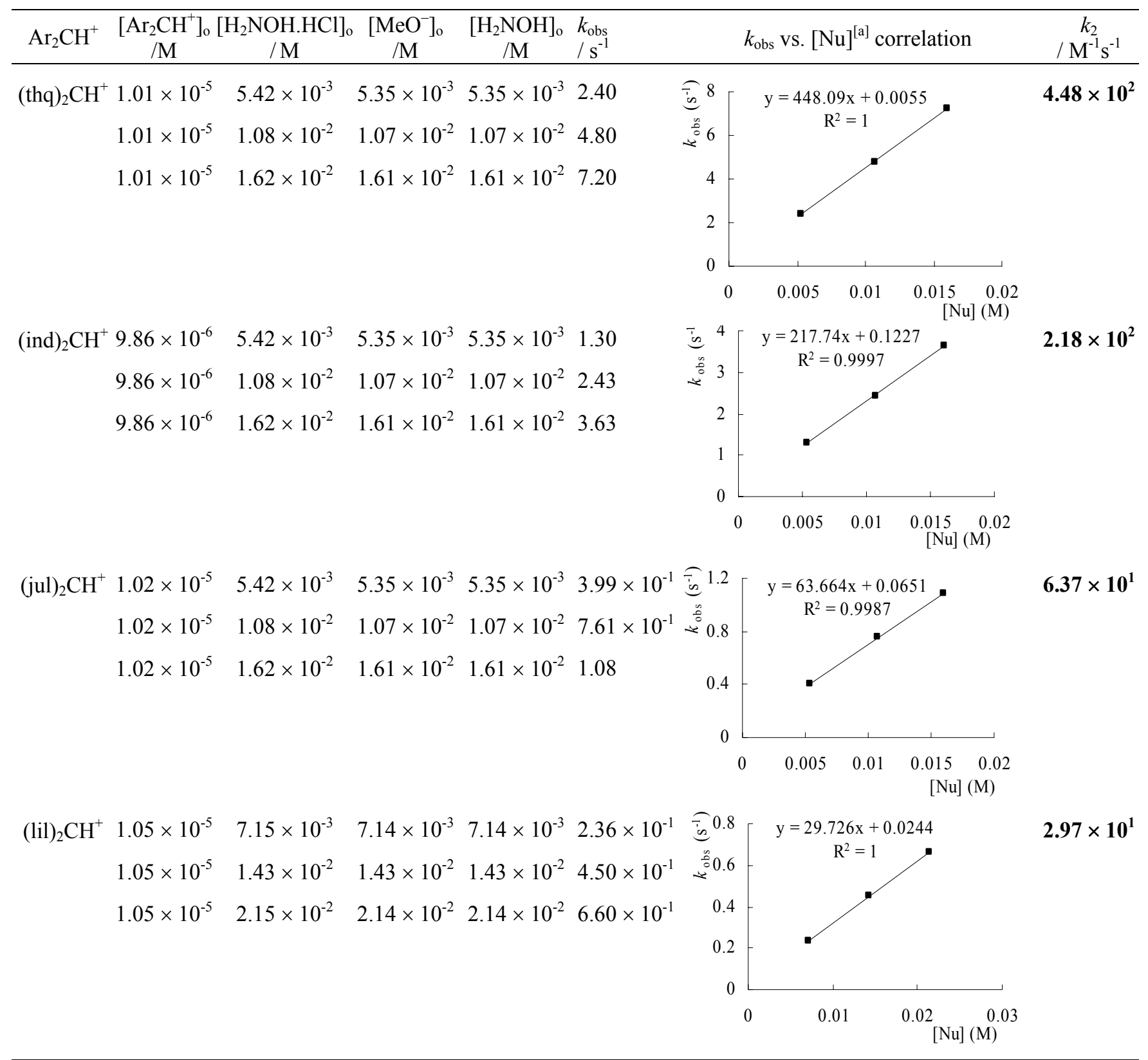

[a] $\mathrm{Nu}=\mathrm{H}_{2} \mathrm{NOH}$, hydroxylamine was freshly generated from hydroxylamine hydrochloride by neutralization with methoxide.

Reactivity parameters: $N=12.23 ; s_{\mathrm{N}}=0.663$

\begin{tabular}{lccc}
\hline Electrophile & $E$ & $k_{2} / \mathrm{M}^{-1} \mathrm{~s}^{-1}$ & $\log k_{2}$ \\
\hline (thq) ${ }_{2} \mathrm{CH}^{+}$ & -8.22 & $4.48 \times 10^{2}$ & 2.65 \\
(ind) ${ }_{2} \mathrm{CH}^{+}$ & -8.76 & $2.18 \times 10^{2}$ & 2.34 \\
(jul) $\mathrm{CH}_{2}$ & -9.45 & $6.37 \times 10^{1}$ & 1.80 \\
(lil $)_{2} \mathrm{CH}^{+}$ & -10.04 & $2.97 \times 10^{1}$ & 1.47 \\
\hline
\end{tabular}

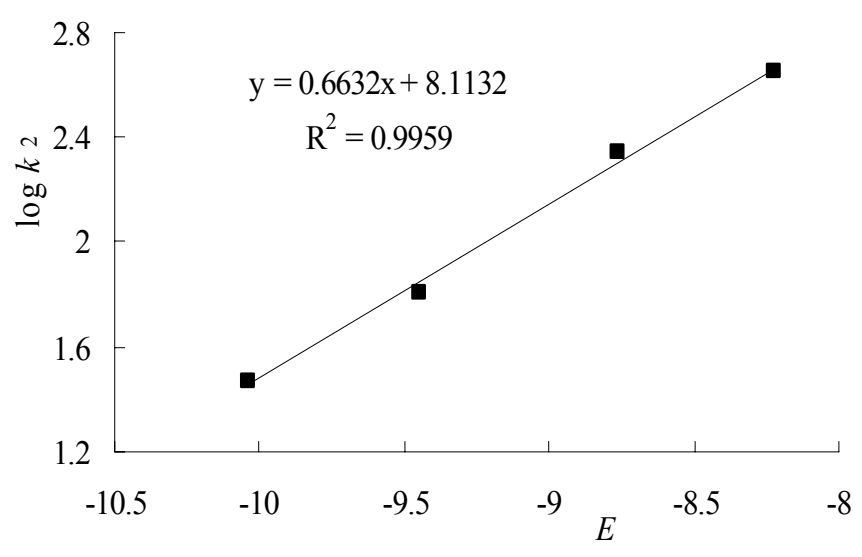




\subsection{Ethanolamine}

Kinetics of the reactions with benzhydrylium ions $\left(20^{\circ} \mathrm{C}\right.$, in $91 / 9$ (v/v) $\mathrm{MeOH} / \mathrm{MeCN}$; stopped-flow)

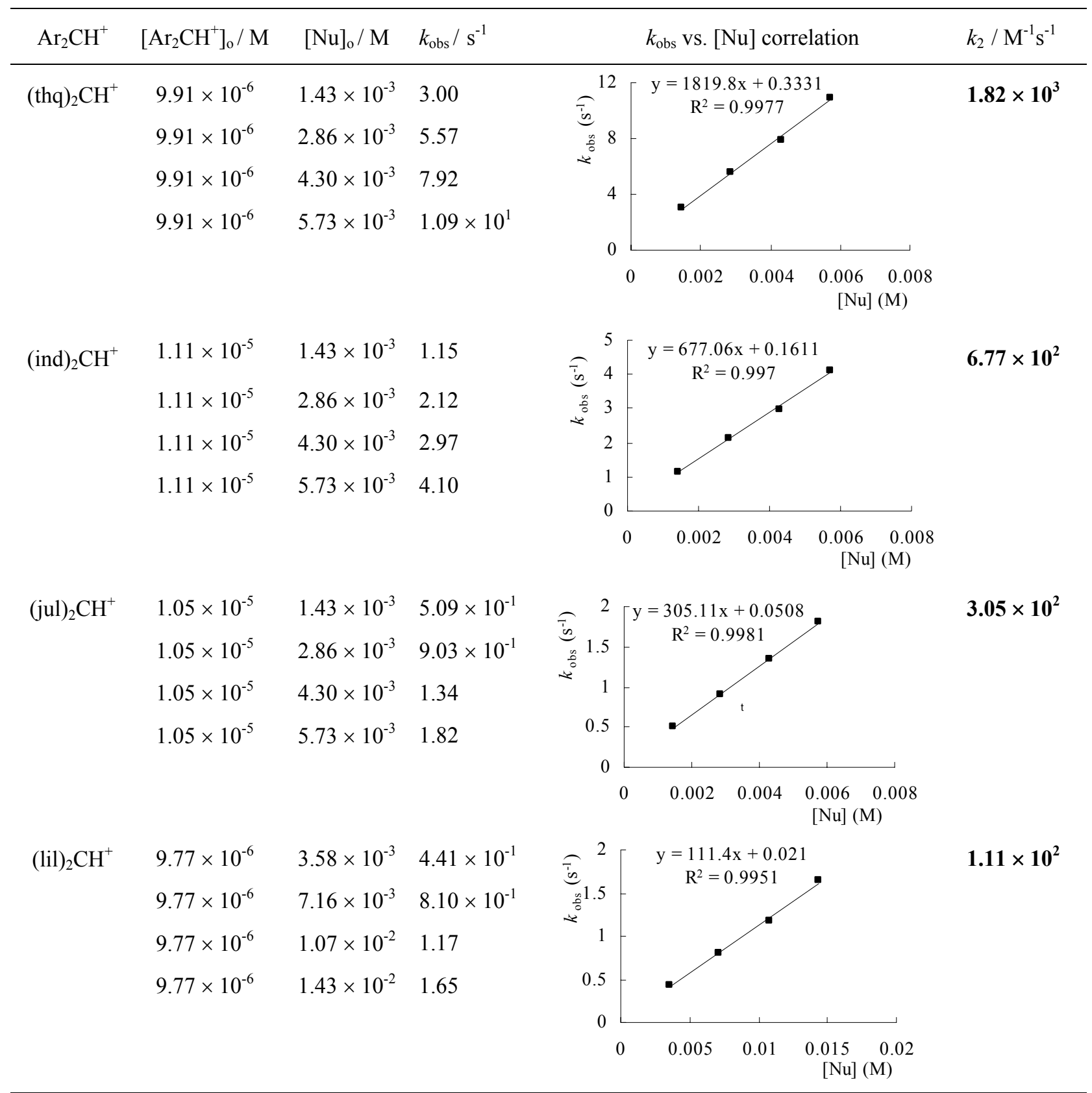

Reactivity parameters: $N=13.23 ; s_{\mathrm{N}}=0.646$

\begin{tabular}{lccc}
\hline Electrophile & $E$ & $k_{2} / \mathrm{M}^{-1} \mathrm{~s}^{-1}$ & $\log k_{2}$ \\
\hline (thq) $)_{2} \mathrm{CH}^{+}$ & -8.22 & $1.82 \times 10^{3}$ & 3.26 \\
(ind) ${ }_{2} \mathrm{CH}^{+}$ & -8.76 & $6.77 \times 10^{2}$ & 2.83 \\
(jul) ${ }_{2} \mathrm{CH}^{+}$ & -9.45 & $3.05 \times 10^{2}$ & 2.48 \\
(lil $)_{2} \mathrm{CH}^{+}$ & -10.04 & $1.11 \times 10^{2}$ & 2.05 \\
\hline
\end{tabular}

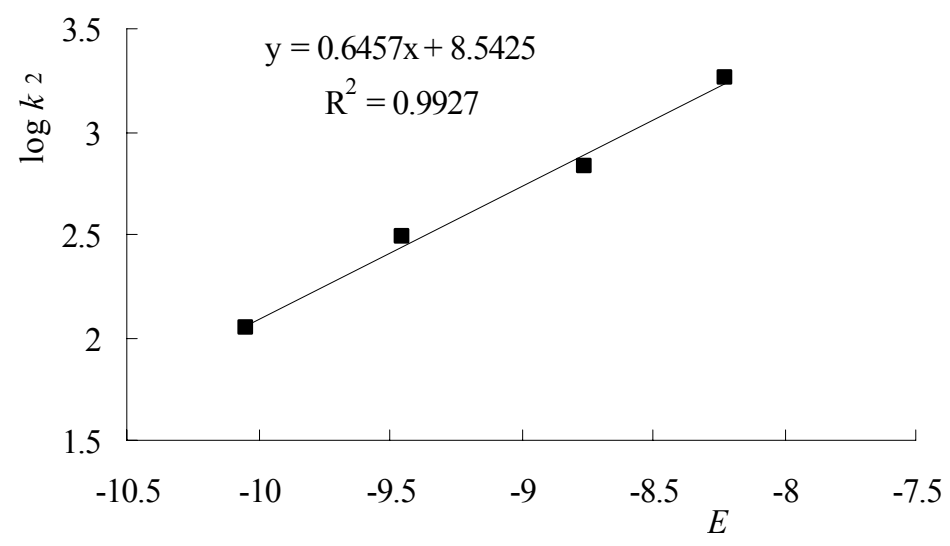




\section{6. $\quad n$-Propylamine}

Kinetics of the reactions with benzhydrylium ions $\left(20^{\circ} \mathrm{C}\right.$, in $91 / 9$ (v/v) $\mathrm{MeOH} / \mathrm{MeCN}$; stopped-flow)

\begin{tabular}{|c|c|c|c|c|c|c|}
\hline $\mathrm{Ar}_{2} \mathrm{CH}^{+}$ & {$\left[\mathrm{Ar}_{2} \mathrm{CH}^{+}\right]_{\mathrm{o}} / \mathrm{M}$} & {$[\mathrm{Nu}]_{\mathrm{o}} / \mathrm{M}$} & $k_{\mathrm{obs}} / \mathrm{s}^{-1}$ & & $k_{\mathrm{obs}}$ vs. $[\mathrm{Nu}]$ correlation & $k_{2} / \mathrm{M}^{-1} \mathrm{~s}^{-1}$ \\
\hline \multirow[t]{5}{*}{ (thq $)_{2} \mathrm{CH}^{+}$} & $1.15 \times 10^{-5}$ & $5.52 \times 10^{-4}$ & 2.06 & क्षृ 8 & \multirow{2}{*}{$\begin{array}{c}y=2889.3 x+0.3894 \\
R^{2}=0.9983\end{array}$} & \multirow[t]{5}{*}{$2.89 \times 10^{3}$} \\
\hline & $1.15 \times 10^{-5}$ & $1.10 \times 10^{-3}$ & 3.46 & $\stackrel{0}{=} 6$ & & \\
\hline & $1.15 \times 10^{-5}$ & $1.66 \times 10^{-3}$ & 5.20 & 4 & & \\
\hline & $1.15 \times 10^{-5}$ & $2.21 \times 10^{-3}$ & 6.80 & 2 & & \\
\hline & & & & & $\begin{array}{c}0.002 \quad 0.003 \\
{[\mathrm{Nu}](\mathrm{M})}\end{array}$ & \\
\hline \multirow[t]{5}{*}{ (ind) ${ }_{2} \mathrm{CH}^{+}$} & $1.03 \times 10^{-5}$ & $5.52 \times 10^{-4}$ & $6.60 \times 10^{-1}$ & $\widehat{[}_{n}^{2} 2.5$ & {$\left[\begin{array}{c}\mathrm{y}=970.38 \mathrm{x}+0.1452 \\
\mathrm{R}^{2}=0.9965\end{array}\right.$} & \multirow[t]{5}{*}{$9.70 \times 10^{2}$} \\
\hline & $1.03 \times 10^{-5}$ & $1.10 \times 10^{-3}$ & 1.22 & $\begin{array}{l}1.5 \\
2\end{array}$ & & \\
\hline & $1.03 \times 10^{-5}$ & $1.66 \times 10^{-3}$ & 1.81 & 1 & & \\
\hline & $1.03 \times 10^{-5}$ & $2.21 \times 10^{-3}$ & 2.25 & 0.5 & & \\
\hline & & & & & $\begin{array}{c}0.002 \quad 0.003 \\
{[\mathrm{Nu}](\mathrm{M})}\end{array}$ & \\
\hline \multirow[t]{5}{*}{$(\mathrm{jul})_{2} \mathrm{CH}^{+}$} & $1.00 \times 10^{-5}$ & $5.52 \times 10^{-4}$ & $2.80 \times 10^{-1}$ & \multirow{5}{*}{ 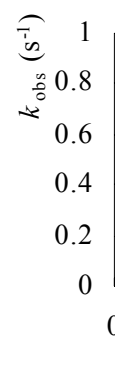 } & \multirow{4}{*}{$\begin{array}{c}\mathrm{y}=401.17 \mathrm{x}+0.0656 \\
\mathrm{R}^{2}=0.9993\end{array}$} & \multirow[t]{5}{*}{$4.01 \times 10^{2}$} \\
\hline & $1.00 \times 10^{-5}$ & $1.10 \times 10^{-3}$ & $5.20 \times 10^{-1}$ & & & \\
\hline & $1.00 \times 10^{-5}$ & $1.66 \times 10^{-3}$ & $7.30 \times 10^{-1}$ & & & \\
\hline & $1.00 \times 10^{-5}$ & $2.21 \times 10^{-3}$ & $9.49 \times 10^{-1}$ & & & \\
\hline & & & & & $\begin{array}{c}0.002 \quad 0.003 \\
{[\mathrm{Nu}](\mathrm{M})}\end{array}$ & \\
\hline \multirow[t]{5}{*}{$(\mathrm{lil})_{2} \mathrm{CH}^{+}$} & $1.01 \times 10^{-5}$ & $5.52 \times 10^{-4}$ & $1.14 \times 10^{-1}$ & \multirow{5}{*}{ 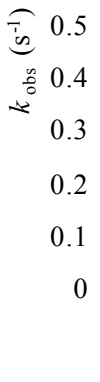 } & $y=172.23 x+0.0231$ & \multirow[t]{5}{*}{$1.72 \times 10^{2}$} \\
\hline & $1.01 \times 10^{-5}$ & $1.10 \times 10^{-3}$ & $2.19 \times 10^{-1}$ & & & \\
\hline & $1.01 \times 10^{-5}$ & $1.66 \times 10^{-3}$ & $3.10 \times 10^{-1}$ & & & \\
\hline & $1.01 \times 10^{-5}$ & $2.21 \times 10^{-3}$ & $4.01 \times 10^{-1}$ & & & \\
\hline & & & & & $\begin{array}{l}0.002 \quad 0.003 \\
{[\mathrm{Nu}](\mathrm{M})}\end{array}$ & \\
\hline
\end{tabular}

Reactivity parameters: $N=13.41 ; s_{\mathrm{N}}=0.657$

\begin{tabular}{lccc}
\hline Electrophile & $E$ & $k_{2} / \mathrm{M}^{-1} \mathrm{~s}^{-1}$ & $\log k_{2}$ \\
\hline (thq $)_{2} \mathrm{CH}^{+}$ & -8.22 & $2.89 \times 10^{3}$ & 3.46 \\
(ind) ${ }_{2} \mathrm{CH}^{+}$ & -8.76 & $9.70 \times 10^{2}$ & 2.99 \\
$(\text { jul })_{2} \mathrm{CH}^{+}$ & -9.45 & $4.01 \times 10^{2}$ & 2.60 \\
$(\text { lil })_{2} \mathrm{CH}^{+}$ & -10.04 & $1.72 \times 10^{2}$ & 2.24 \\
\hline
\end{tabular}

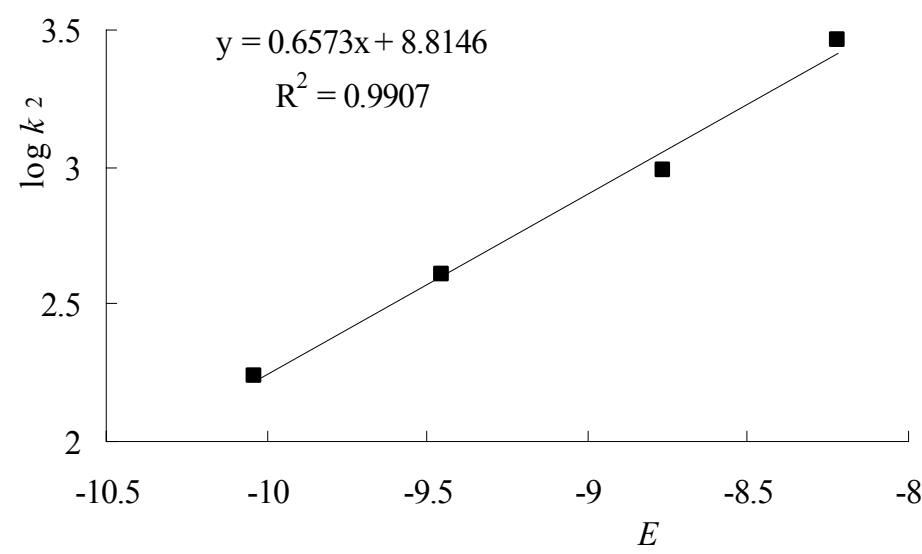




\subsection{Benzylamine}

Kinetics of the reactions with benzhydrylium ions $\left(20^{\circ} \mathrm{C}\right.$, in $91 / 9$ (v/v) $\mathrm{MeOH} / \mathrm{MeCN}$; stopped-flow)

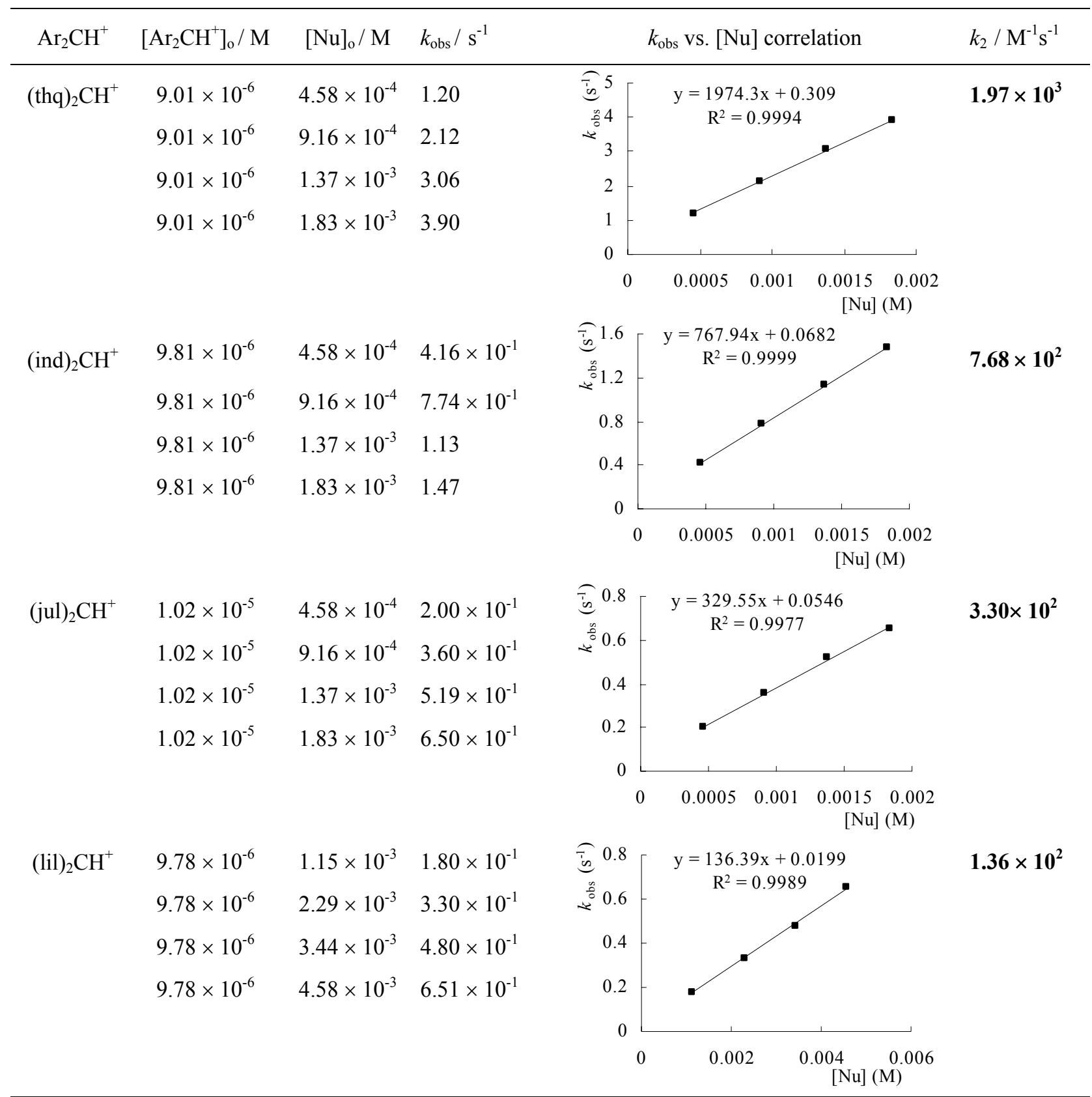

Reactivity parameters: $N=13.46 ; s_{\mathrm{N}}=0.624$

\begin{tabular}{lccc}
\hline Electrophile & $E$ & $k_{2} / \mathrm{M}^{-1} \mathrm{~s}^{-1}$ & $\log k_{2}$ \\
\hline (thq $)_{2} \mathrm{CH}^{+}$ & -8.22 & $1.97 \times 10^{3}$ & 3.30 \\
(ind $)_{2} \mathrm{CH}^{+}$ & -8.76 & $7.68 \times 10^{2}$ & 2.89 \\
(jul) ${ }_{2} \mathrm{CH}^{+}$ & -9.45 & $3.30 \times 10^{2}$ & 2.52 \\
$(\text { lil })_{2} \mathrm{CH}^{+}$ & -10.04 & $1.36 \times 10^{2}$ & 2.13 \\
\hline
\end{tabular}

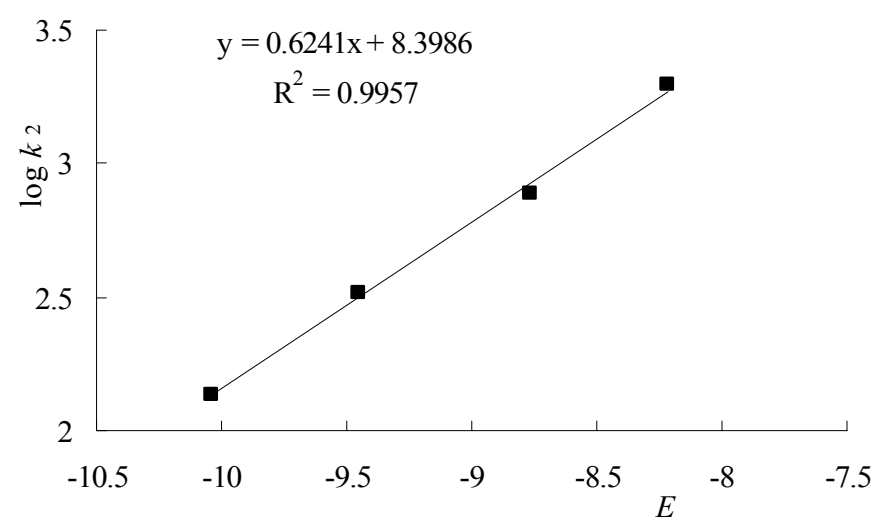




\subsection{Hydrazine}

Kinetics of the reactions with benzhydrylium ions $\left(20^{\circ} \mathrm{C}\right.$, in $91 / 9$ (v/v) $\mathrm{MeOH} / \mathrm{MeCN}$; stopped-flow)

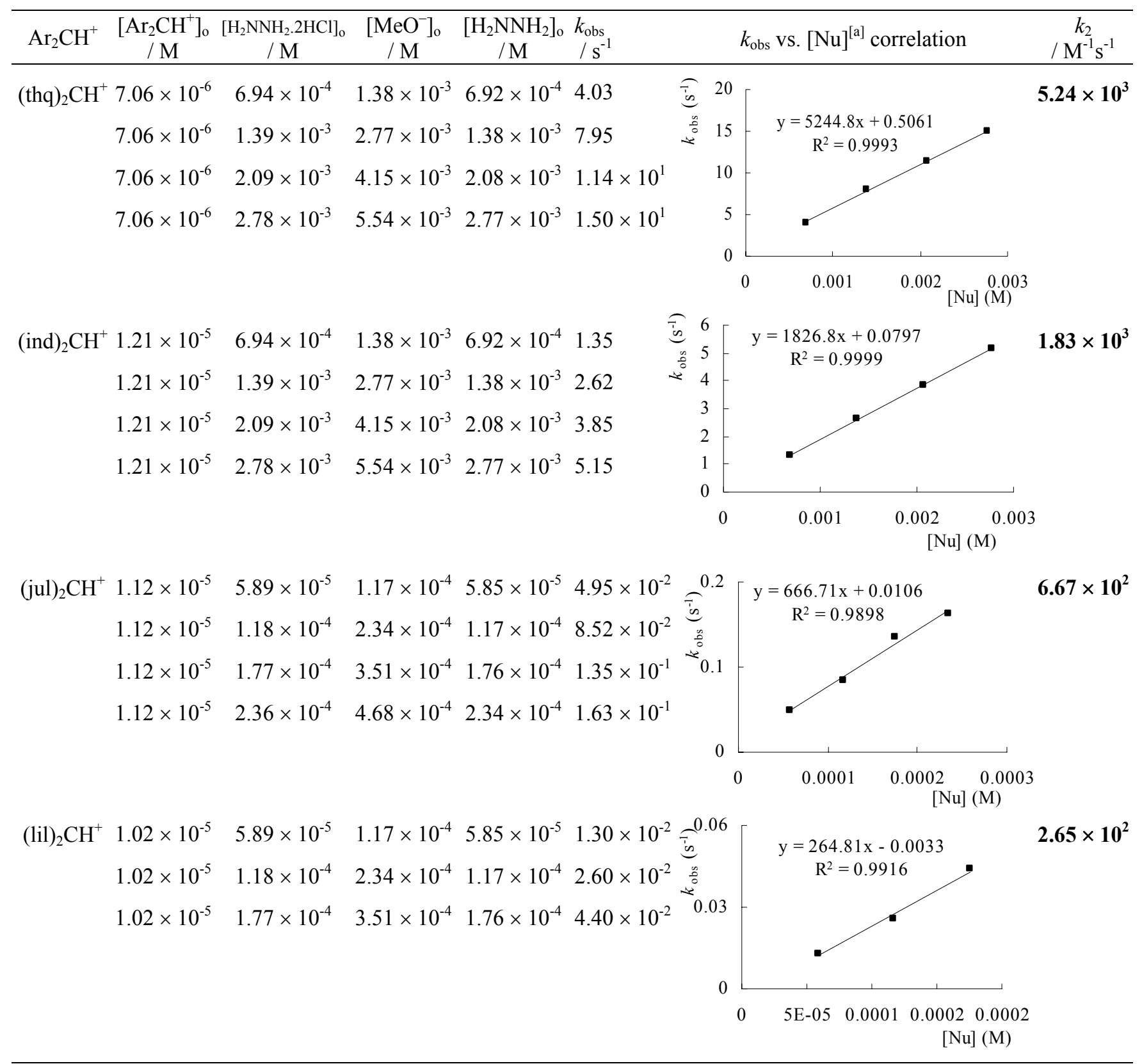

[a] $\mathrm{Nu}=\mathrm{H}_{2} \mathrm{NNH}_{2}$, hydrazine was freshly generated from hydrazine dihydrochloride by neutralization with methoxide.

Reactivity parameters: $N=13.47 ; s_{\mathrm{N}}=0.702$

\begin{tabular}{lccc}
\hline Electrophile & $E$ & $k_{2} / \mathrm{M}^{-1} \mathrm{~s}^{-1}$ & $\log k_{2}$ \\
\hline (thq $)_{2} \mathrm{CH}^{+}$ & -8.22 & $5.24 \times 10^{3}$ & 3.72 \\
(ind) ${ }_{2} \mathrm{CH}^{+}$ & -8.76 & $1.83 \times 10^{3}$ & 3.26 \\
(jul) $\mathrm{CH}^{+}$ & -9.45 & $6.67 \times 10^{2}$ & 2.82 \\
$(\mathrm{lil})_{2} \mathrm{CH}^{+}$ & -10.04 & $2.65 \times 10^{2}$ & 2.42 \\
\hline
\end{tabular}

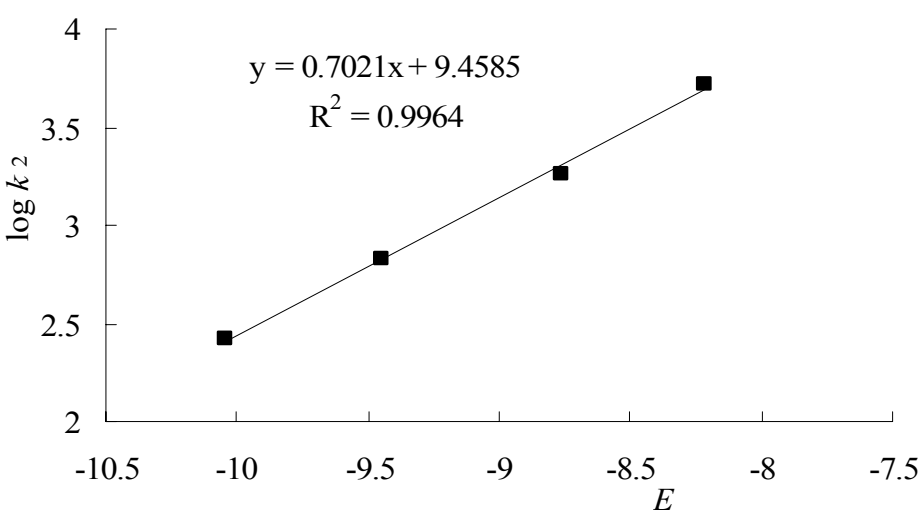




\subsection{Diethanolamine}

Kinetics of the reactions with benzhydrylium ions $\left(20^{\circ} \mathrm{C}\right.$, in $91 / 9$ (v/v) $\mathrm{MeOH} / \mathrm{MeCN}$; stopped-flow)

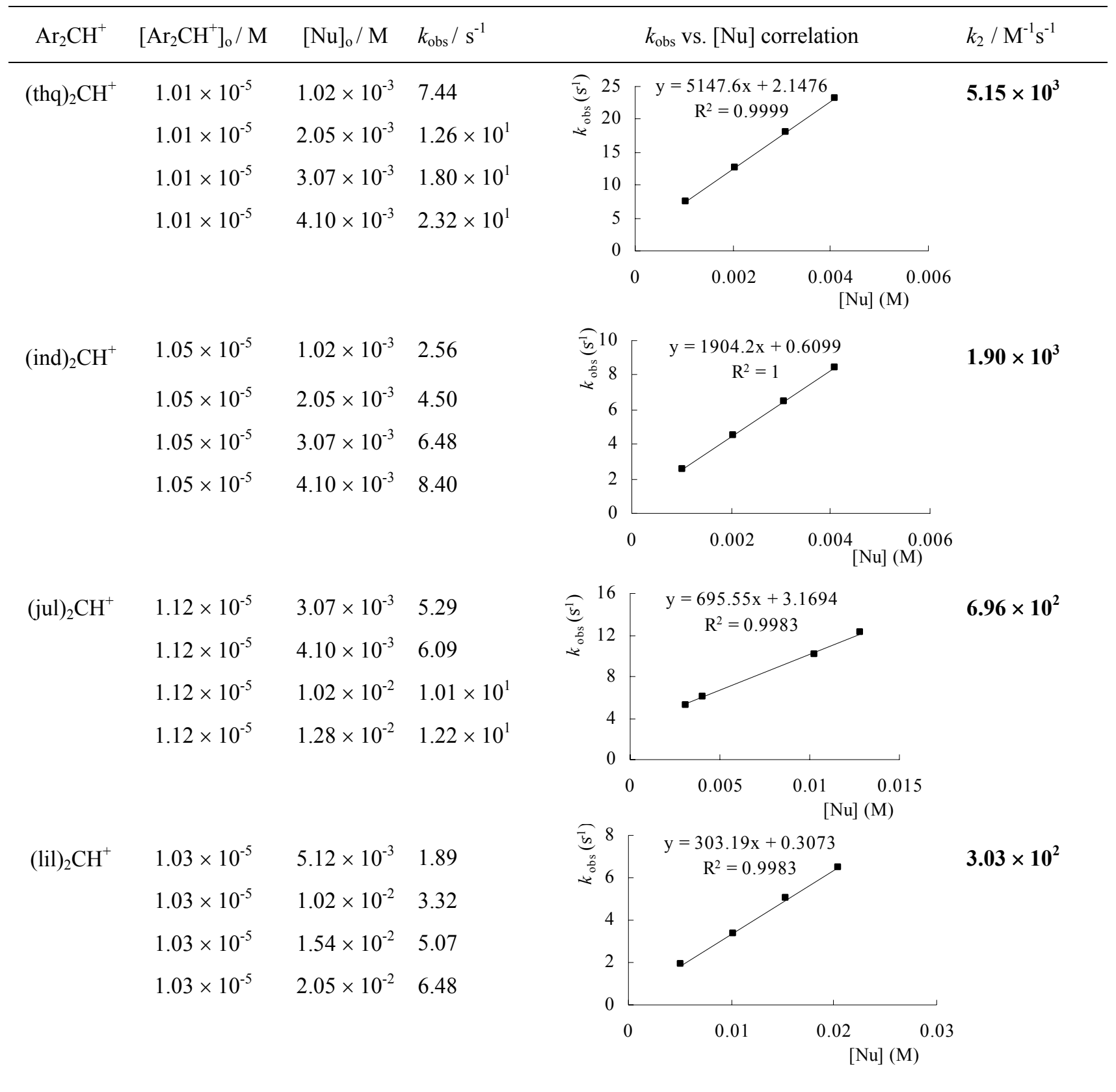

Reactivity parameters: $N=13.71 ; s_{\mathrm{N}}=0.670$

\begin{tabular}{lccc}
\hline Electrophile & $E$ & $k_{2} / \mathrm{M}^{-1} \mathrm{~s}^{-1}$ & $\log k_{2}$ \\
\hline (thq) ${ }_{2} \mathrm{CH}^{+}$ & -8.22 & $5.15 \times 10^{3}$ & 3.71 \\
(ind) ${ }_{2} \mathrm{CH}^{+}$ & -8.76 & $1.90 \times 10^{3}$ & 3.28 \\
(jul) $\mathrm{CH}^{+}$ & -9.45 & $6.96 \times 10^{2}$ & 2.84 \\
$(\mathrm{lil}) \mathrm{CH}^{+}$ & -10.04 & $3.03 \times 10^{2}$ & 2.48 \\
\hline
\end{tabular}

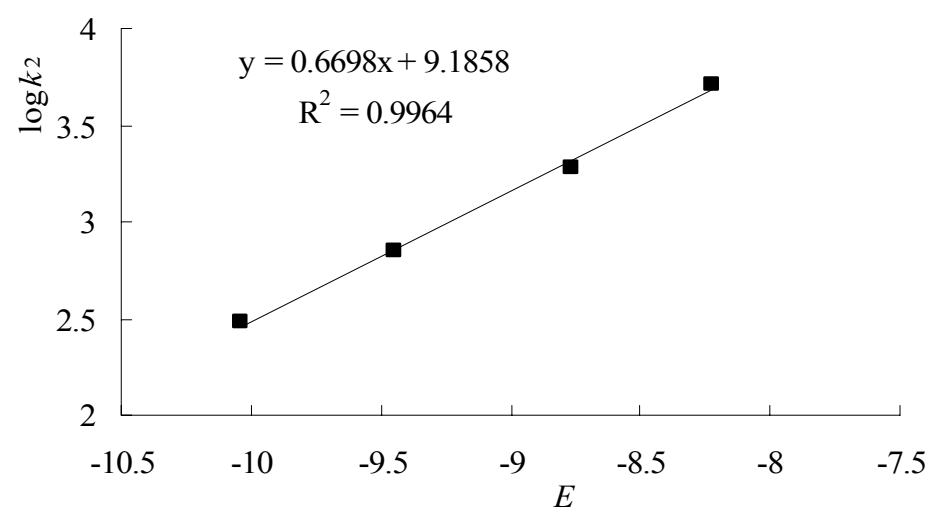




\subsection{Morpholine}

Kinetics of the reactions with benzhydrylium ions $\left(20^{\circ} \mathrm{C}\right.$, in $91 / 9$ (v/v) $\mathrm{MeOH} / \mathrm{MeCN}$; stopped-flow)

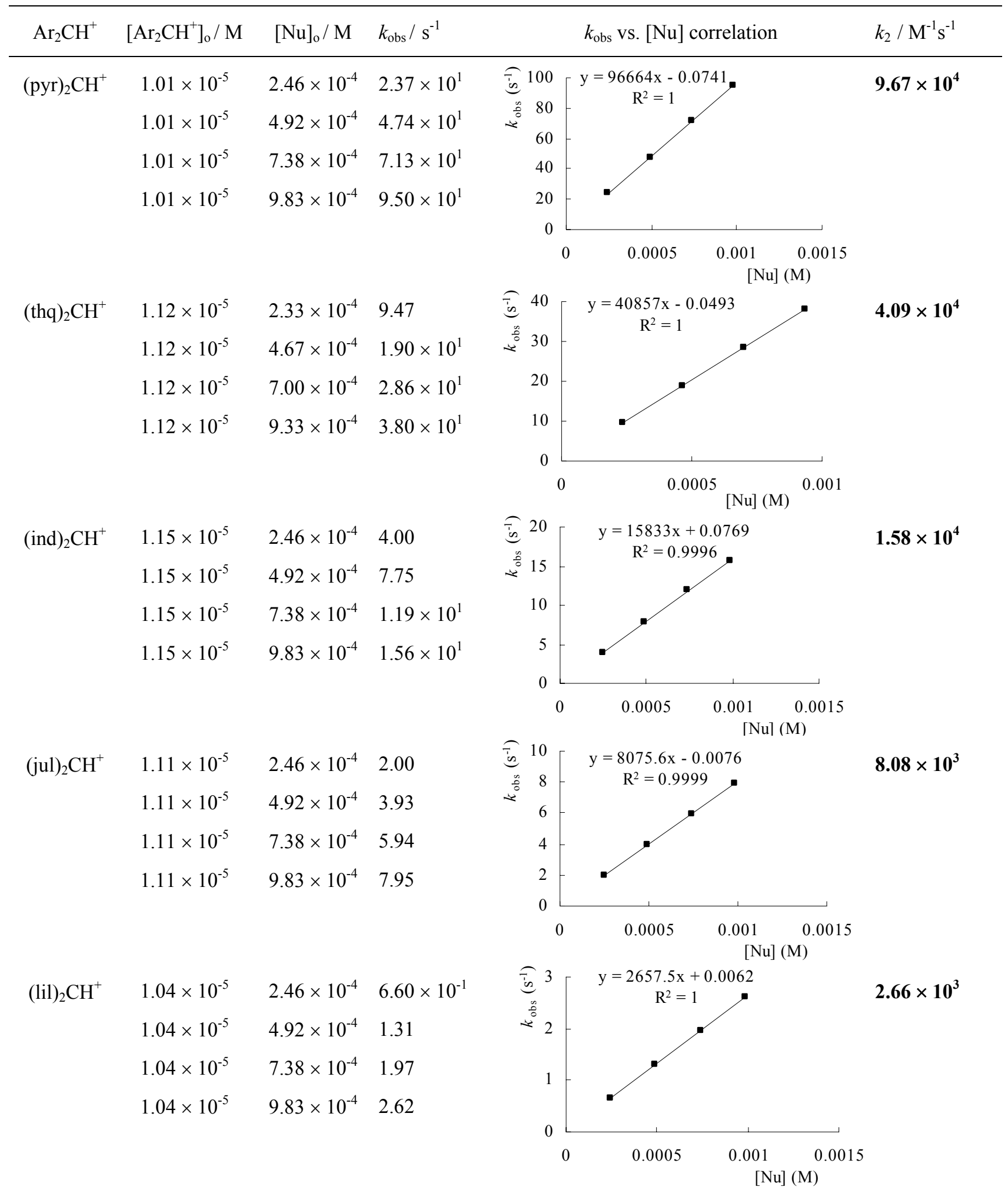


Reactivity parameters: $N=15.40 ; s_{\mathrm{N}}=0.643$

\begin{tabular}{lccc}
\hline Electrophile & $E$ & $k_{2} / \mathrm{M}^{-1} \mathrm{~s}^{-1}$ & $\log k_{2}$ \\
\hline (pyr) ${ }_{2} \mathrm{CH}^{+}$ & -7.69 & $9.67 \times 10^{4}$ & 4.99 \\
(thq $)_{2} \mathrm{CH}^{+}$ & -8.22 & $4.09 \times 10^{4}$ & 4.61 \\
(ind $)_{2} \mathrm{CH}^{+}$ & -8.76 & $1.58 \times 10^{4}$ & 4.20 \\
$(\text { jul })_{2} \mathrm{CH}^{+}$ & -9.45 & $8.08 \times 10^{3}$ & 3.91 \\
$(\text { lil })_{2} \mathrm{CH}^{+}$ & -10.04 & $2.66 \times 10^{3}$ & 3.42 \\
\hline
\end{tabular}

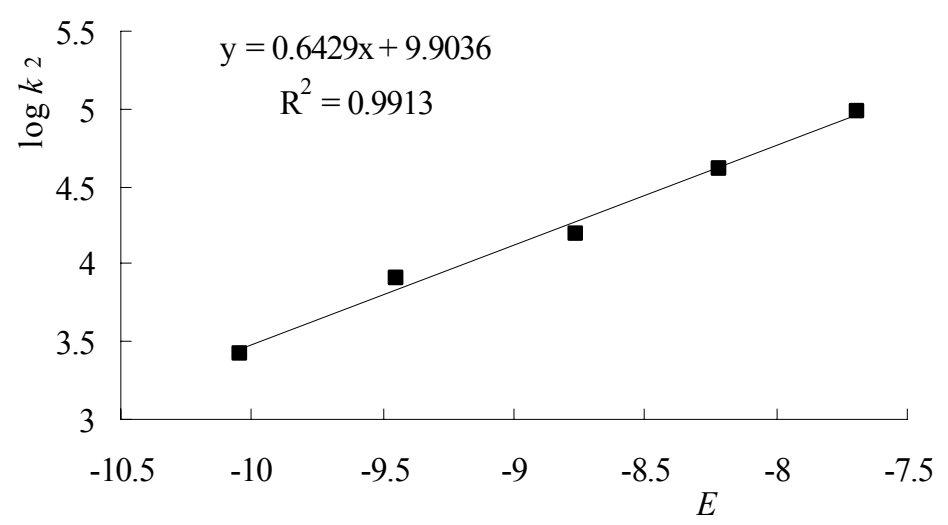

\subsection{Piperidine}

Kinetics of the reactions with benzhydrylium ions $\left(20^{\circ} \mathrm{C}\right.$, in $91 / 9$ (v/v) $\mathrm{MeOH} / \mathrm{MeCN}$; stopped-flow)

\begin{tabular}{|c|c|c|c|c|c|c|}
\hline $\mathrm{Ar}_{2} \mathrm{CH}^{+}$ & {$\left[\mathrm{Ar}_{2} \mathrm{CH}^{+}\right]_{\mathrm{o}} / \mathrm{M}$} & {$[\mathrm{Nu}]_{\mathrm{o}} / \mathrm{M}$} & $k_{\mathrm{obs}} / \mathrm{s}^{-1}$ & & $k_{\text {obs }}$ vs. $[\mathrm{Nu}]$ correlation & $k_{2} / \mathrm{M}^{-1} \mathrm{~s}^{-1}$ \\
\hline \multirow[t]{5}{*}{$(\text { pyr })_{2} \mathrm{CH}^{+}$} & $1.01 \times 10^{-5}$ & $2.15 \times 10^{-4}$ & $2.92 \times 10^{1}$ & $\underbrace{\hat{e}_{0}^{\infty}}_{\infty} 160$ & $y=137902 x-0.1321$ & $1.38 \times 10^{5}$ \\
\hline & $1.01 \times 10^{-5}$ & $4.30 \times 10^{-4}$ & $5.94 \times 10^{1}$ & $\stackrel{1}{-1} 120$ & & \\
\hline & $1.01 \times 10^{-5}$ & $6.45 \times 10^{-4}$ & $8.92 \times 10^{1}$ & 80 & & \\
\hline & $1.01 \times 10^{-5}$ & $8.60 \times 10^{-4}$ & $1.18 \times 10^{2}$ & 40 & & \\
\hline & & & & 0 & $0.0005[\mathrm{Nu}](\mathrm{M}){ }^{0.001}$ & \\
\hline \multirow[t]{5}{*}{ (thq $)_{2} \mathrm{CH}^{+}$} & $1.11 \times 10^{-5}$ & $2.15 \times 10^{-4}$ & $1.26 \times 10^{1}$ & 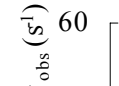 & $\begin{array}{r}y=59157 x-0.0 \\
R^{2}=1\end{array}$ & $5.92 \times 10^{4}$ \\
\hline & $1.11 \times 10^{-5}$ & $4.29 \times 10^{-4}$ & $2.54 \times 10^{1}$ & 40 & & \\
\hline & $1.11 \times 10^{-5}$ & $6.44 \times 10^{-4}$ & $3.80 \times 10^{1}$ & 20 & & \\
\hline & $1.11 \times 10^{-5}$ & $8.58 \times 10^{-4}$ & $5.07 \times 10^{1}$ & & & \\
\hline & & & & 0 & $0.0005[\mathrm{Nu}](\mathrm{M}) \quad 0.001$ & \\
\hline \multirow[t]{5}{*}{ (ind) ${ }_{2} \mathrm{CH}^{+}$} & $1.05 \times 10^{-5}$ & $2.15 \times 10^{-4}$ & 4.80 & 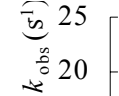 & $\begin{array}{c}\mathrm{y}=22603 \mathrm{x}-0.0089 \\
\mathrm{R}^{2}=0.9999\end{array}$ & $2.26 \times 10^{4}$ \\
\hline & $1.05 \times 10^{-5}$ & $4.30 \times 10^{-4}$ & 9.78 & 15 & & \\
\hline & $1.05 \times 10^{-5}$ & $6.45 \times 10^{-4}$ & $1.46 \times 10^{1}$ & 10 & & \\
\hline & $1.05 \times 10^{-5}$ & $8.60 \times 10^{-4}$ & $1.94 \times 10^{1}$ & 5 & & \\
\hline & & & & 0 & $0.0005\left[\mathrm{Nu}{ }_{(\mathrm{M})} 0.001\right.$ & \\
\hline \multirow[t]{5}{*}{$(\mathrm{jul})_{2} \mathrm{CH}^{+}$} & $1.15 \times 10^{-5}$ & $2.15 \times 10^{-4}$ & 2.39 & 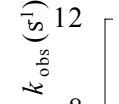 & $\begin{array}{c}y=11183 x-0.0341 \\
R^{2}=1\end{array}$ & $1.12 \times 10^{4}$ \\
\hline & $1.15 \times 10^{-5}$ & $4.30 \times 10^{-4}$ & 4.75 & & & \\
\hline & $1.15 \times 10^{-5}$ & $6.45 \times 10^{-4}$ & 7.17 & 4 & & \\
\hline & $1.15 \times 10^{-5}$ & $8.60 \times 10^{-4}$ & 9.60 & 0 & & \\
\hline & & & & 0 & $0.0005[\mathrm{Nu}](\mathrm{M}) 0.001$ & \\
\hline
\end{tabular}




\section{Continued}

\begin{tabular}{|c|c|c|c|c|c|c|}
\hline $\mathrm{Ar}_{2} \mathrm{CH}^{+}$ & {$\left[\mathrm{Ar}_{2} \mathrm{CH}^{+}\right]_{\mathrm{o}} / \mathrm{M}$} & {$[\mathrm{Nu}]_{\mathrm{o}} / \mathrm{M}$} & $k_{\mathrm{obs}} / \mathrm{s}^{-1}$ & & $k_{\text {obs }}$ vs. $[\mathrm{Nu}]$ correlation & $k_{2} / \mathrm{M}^{-1} \mathrm{~s}^{-1}$ \\
\hline \multirow[t]{5}{*}{$(\mathrm{lil}){ }_{2} \mathrm{CH}^{+}$} & $1.04 \times 10^{-5}$ & $2.15 \times 10^{-4}$ & $8.49 \times 10^{-1}$ & 盾 4 & \multirow{4}{*}{$\begin{array}{c}\mathrm{y}=3843.7 \mathrm{x}-0.0008 \\
\mathrm{R}^{2}=0.9988\end{array}$} & \multirow[t]{4}{*}{$3.84 \times 10^{3}$} \\
\hline & $1.04 \times 10^{-5}$ & $4.30 \times 10^{-4}$ & 1.60 & $\stackrel{0}{-2} 3$ & & \\
\hline & $1.04 \times 10^{-5}$ & $6.45 \times 10^{-4}$ & 2.51 & 2 & & \\
\hline & \multirow[t]{2}{*}{$1.04 \times 10^{-5}$} & $8.60 \times 10^{-4}$ & 3.30 & 1 & & \\
\hline & & & & 0 & $0.0005[\mathrm{Nu}](\mathrm{M}){ }^{0.001}$ & \\
\hline
\end{tabular}

Reactivity parameters: $N=15.63 ; s_{\mathrm{N}}=0.644$

\begin{tabular}{lccc}
\hline Electrophile & $E$ & $k_{2} / \mathrm{M}^{-1} \mathrm{~s}^{-1}$ & $\log k_{2}$ \\
\hline (pyr) ${ }_{2} \mathrm{CH}^{+}$ & -7.69 & $1.38 \times 10^{5}$ & 5.14 \\
(thq) $)_{2} \mathrm{CH}^{+}$ & -8.22 & $5.92 \times 10^{4}$ & 4.77 \\
(ind) ${ }_{2} \mathrm{CH}^{+}$ & -8.76 & $2.26 \times 10^{4}$ & 4.35 \\
(jul) $\mathrm{CH}^{+}$ & -9.45 & $1.12 \times 10^{4}$ & 4.05 \\
$(\mathrm{lil})_{2} \mathrm{CH}^{+}$ & -10.04 & $3.84 \times 10^{3}$ & 3.58 \\
\hline
\end{tabular}

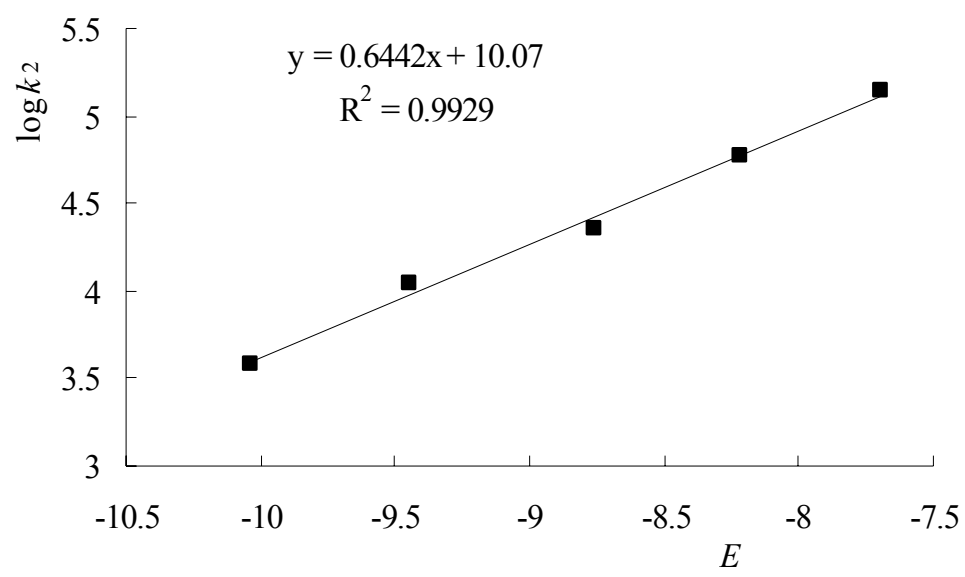

\subsection{Pyrrolidine}

Kinetics of the reactions with benzhydrylium ions $\left(20^{\circ} \mathrm{C}\right.$, in $91 / 9$ (v/v) $\mathrm{MeOH} / \mathrm{MeCN}$; stopped-flow)

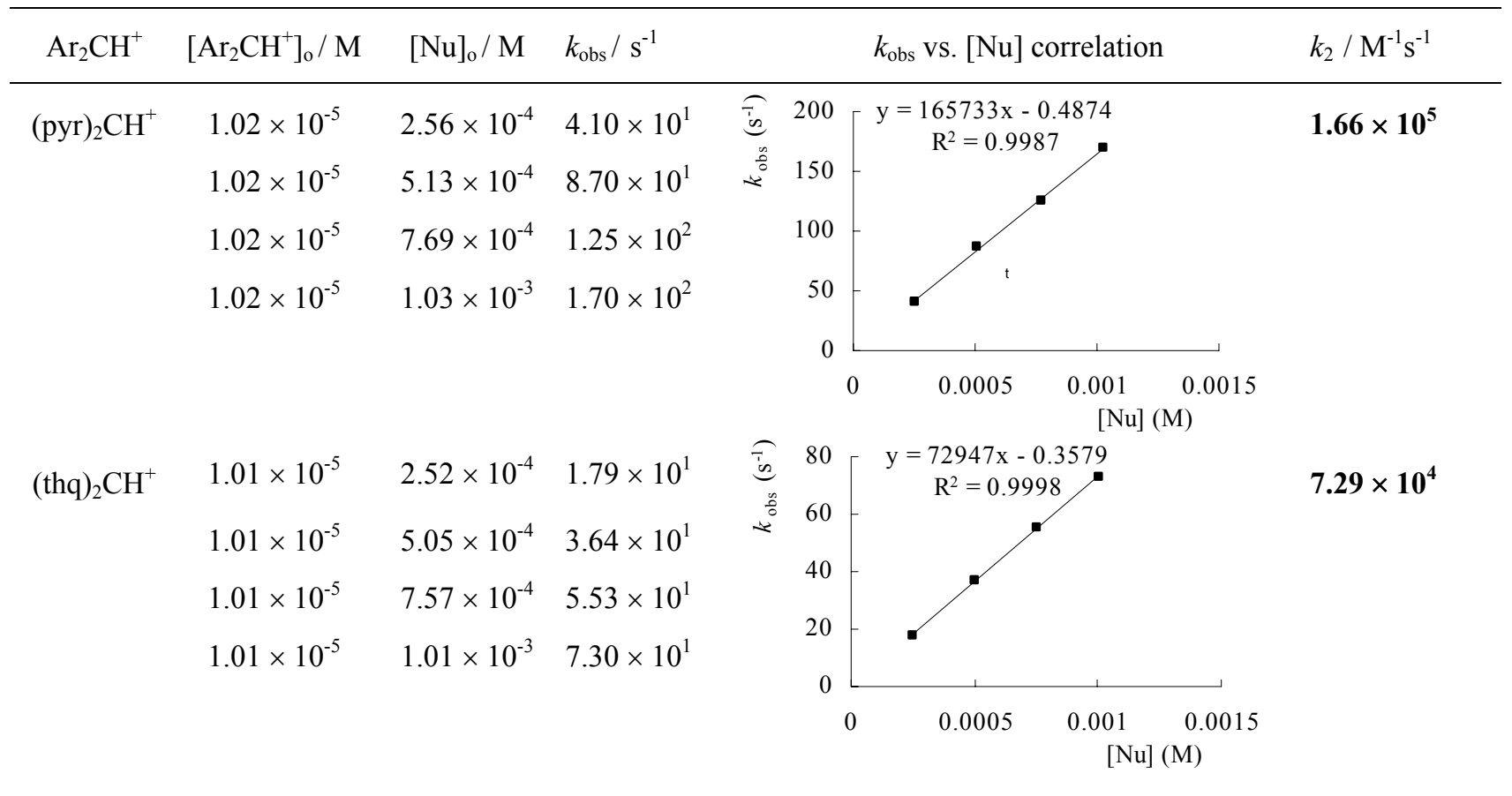




\begin{tabular}{|c|c|c|c|c|c|c|}
\hline $\mathrm{Ar}_{2} \mathrm{CH}^{+}$ & {$\left[\mathrm{Ar}_{2} \mathrm{CH}^{+}\right]_{\mathrm{o}} / \mathrm{M}$} & {$[\mathrm{Nu}]_{0} / \mathrm{M}$} & $k_{\mathrm{obs}} / \mathrm{s}^{-1}$ & & $k_{\text {obs }}$ vs. $[\mathrm{Nu}]$ correlation & $k_{2} / \mathrm{M}^{-1} \mathrm{~s}^{-1}$ \\
\hline \multirow[t]{5}{*}{ (ind) ${ }_{2} \mathrm{CH}^{+}$} & $9.92 \times 10^{-6}$ & $2.56 \times 10^{-4}$ & 7.00 & \multirow{5}{*}{ 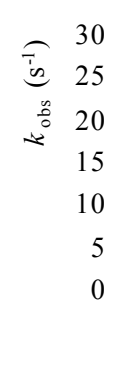 } & $\begin{array}{c}\mathrm{y}=27746 \mathrm{x}-0.0021 \\
\mathrm{R}^{2}=0.9997\end{array}$ & \multirow[t]{5}{*}{$2.77 \times 10^{4}$} \\
\hline & $9.92 \times 10^{-6}$ & $5.13 \times 10^{-4}$ & $1.45 \times 10^{1}$ & & & \\
\hline & $9.92 \times 10^{-6}$ & $7.69 \times 10^{-4}$ & $2.12 \times 10^{1}$ & & & \\
\hline & $9.92 \times 10^{-6}$ & $1.03 \times 10^{-3}$ & $2.85 \times 10^{1}$ & & & \\
\hline & & & & & $\begin{array}{c}0.001 \quad 0.0015 \\
{[\mathrm{Nu}](\mathrm{M})}\end{array}$ & \\
\hline$(\mathrm{jul})_{2} \mathrm{CH}^{+}$ & $1.05 \times 10^{-5}$ & $2.56 \times 10^{-4}$ & 3.62 & $\widehat{F}^{20}$ & $\begin{array}{c}y=14212 x-0.0054 \\
R^{2}=0.9998\end{array}$ & $1.42 \times 10^{4}$ \\
\hline & $1.05 \times 10^{-5}$ & $5.13 \times 10^{-4}$ & 7.27 & $\underbrace{\infty}_{\frac{n}{0}} 15$ & 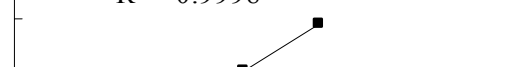 & \\
\hline & $1.05 \times 10^{-5}$ & $7.69 \times 10^{-4}$ & $1.10 \times 10^{1}$ & & & \\
\hline & $1.05 \times 10^{-5}$ & $1.03 \times 10^{-3}$ & $1.45 \times 10^{1}$ & 5 & & \\
\hline & & & & & $\begin{array}{ccc}0.0005 & 0.001 & 0.0015 \\
& {[\mathrm{Nu}](\mathrm{M})}\end{array}$ & \\
\hline$(\mathrm{lil})_{2} \mathrm{CH}^{+}$ & $1.07 \times 10^{-5}$ & $2.56 \times 10^{-4}$ & 1.22 & $\widehat{\hat{\omega}}^{6}$ & $\begin{array}{c}\mathrm{y}=5107 \mathrm{x}-0.0059 \\
\mathrm{R}^{2}=0.9934\end{array}$ & $5.11 \times 10^{3}$ \\
\hline & $1.07 \times 10^{-5}$ & $5.13 \times 10^{-4}$ & 2.65 & $\overbrace{0}^{\frac{n}{0}} 4$ & & \\
\hline & $1.07 \times 10^{-5}$ & $7.69 \times 10^{-4}$ & 4.10 & 2 & & \\
\hline & $1.07 \times 10^{-5}$ & $1.03 \times 10^{-3}$ & 5.10 & & & \\
\hline & & & & ( & $\begin{array}{l}0.0010 .0015 \\
{[\mathrm{Nu}](\mathrm{M})}\end{array}$ & \\
\hline
\end{tabular}

Reactivity parameters: $N=15.97 ; s_{\mathrm{N}}=0.627$

\begin{tabular}{lccc}
\hline Electrophile & $E$ & $k_{2} / \mathrm{M}^{-1} \mathrm{~s}^{-1}$ & $\log k_{2}$ \\
\hline (pyr) ${ }_{2} \mathrm{CH}^{+}$ & -7.69 & $1.66 \times 10^{5}$ & 5.22 \\
(thq $)_{2} \mathrm{CH}^{+}$ & -8.22 & $7.29 \times 10^{4}$ & 4.86 \\
(ind $)_{2} \mathrm{CH}^{+}$ & -8.76 & $2.77 \times 10^{4}$ & 4.44 \\
(jul) ${ }_{2} \mathrm{CH}^{+}$ & -9.45 & $1.42 \times 10^{4}$ & 4.15 \\
$(\mathrm{lil})_{2} \mathrm{CH}^{+}$ & -10.04 & $5.11 \times 10^{3}$ & 3.71 \\
\hline
\end{tabular}

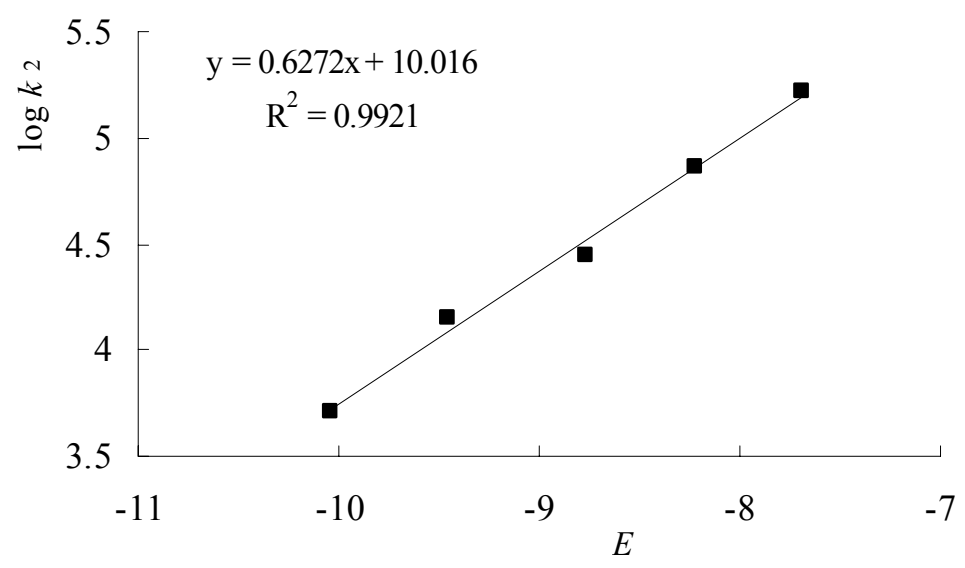




\section{Rate constants for the reactions of the S-methyldibenzothiophenium ion with nucleophiles}

The reactions of the $S$-methyldibenzothiophenium ion with nucleophiles were followed by UV-Vis spectroscopy. ${ }^{[\mathrm{S} 3]}$

The rates of most of the reactions were determined by using a J\&M TIDAS diode array spectrophotometer which was controlled by Labcontrol Spectacle software and connected to a Hellma 661.502-QX quartz Suprasil immersion probe ( $5 \mathrm{~mm}$ light path) via fiber optic cables and standard SMA connectors. The remaining reactions were followed using Hi-Tech SF-61DX2 stopped-flow spectrophotometer systems (controlled by Hi-Tech KinetAsyst2 software).

Because of the reaction of the $S$-methyldibenzothiophenium ion with methanol (used as a solvent), the stock solutions of $S$-methyldibenzothiophenium triflinate were prepared in acetonitrile. Thus, the measurements conducted with the J\&M spectrometer were performed in presence of 1 to $2 \%(\mathrm{v} / \mathrm{v})$ of acetonitrile (100-200 $\mu \mathrm{L}$ acetonitrile per $25 \mathrm{~mL}$ methanol). Experiments with the stopped-flow instrument were performed in presence of $9 \%(\mathrm{v} / \mathrm{v})$ of the co-solvent (10/1 mixing of nucleophile solutions in methanol and electrophile solutions in acetonitrile). Experiments for the reaction of the methoxide anion with the $S$ methyldibenzothiophenium ion showed that a low content of the co-solvent acetonitrile did not influence the rate constant noticeable. Therefore, we considered the rate constants determined in the presence of a small amount of acetonitrile also to be representative for the corresponding rate constants in pure methanol.

The temperature of the solutions during all kinetic studies was kept constant $\left(20 \pm 0.1^{\circ} \mathrm{C}\right)$ by using circulating bath thermostats. 


\subsection{Kinetics of the reactions with different nucleophiles}

$\left(20^{\circ} \mathrm{C}\right.$, in methanol, J\&M spectrometer, evaluated at $\left.271 \mathrm{~nm}\right)$

Plots of $k_{\mathrm{obs}}$ vs. the concentrations of the nucleophiles were linear with slopes corresponding to the secondorder rate constants $k_{2}\left(\mathrm{M}^{-1} \mathrm{~s}^{-1}\right)$.

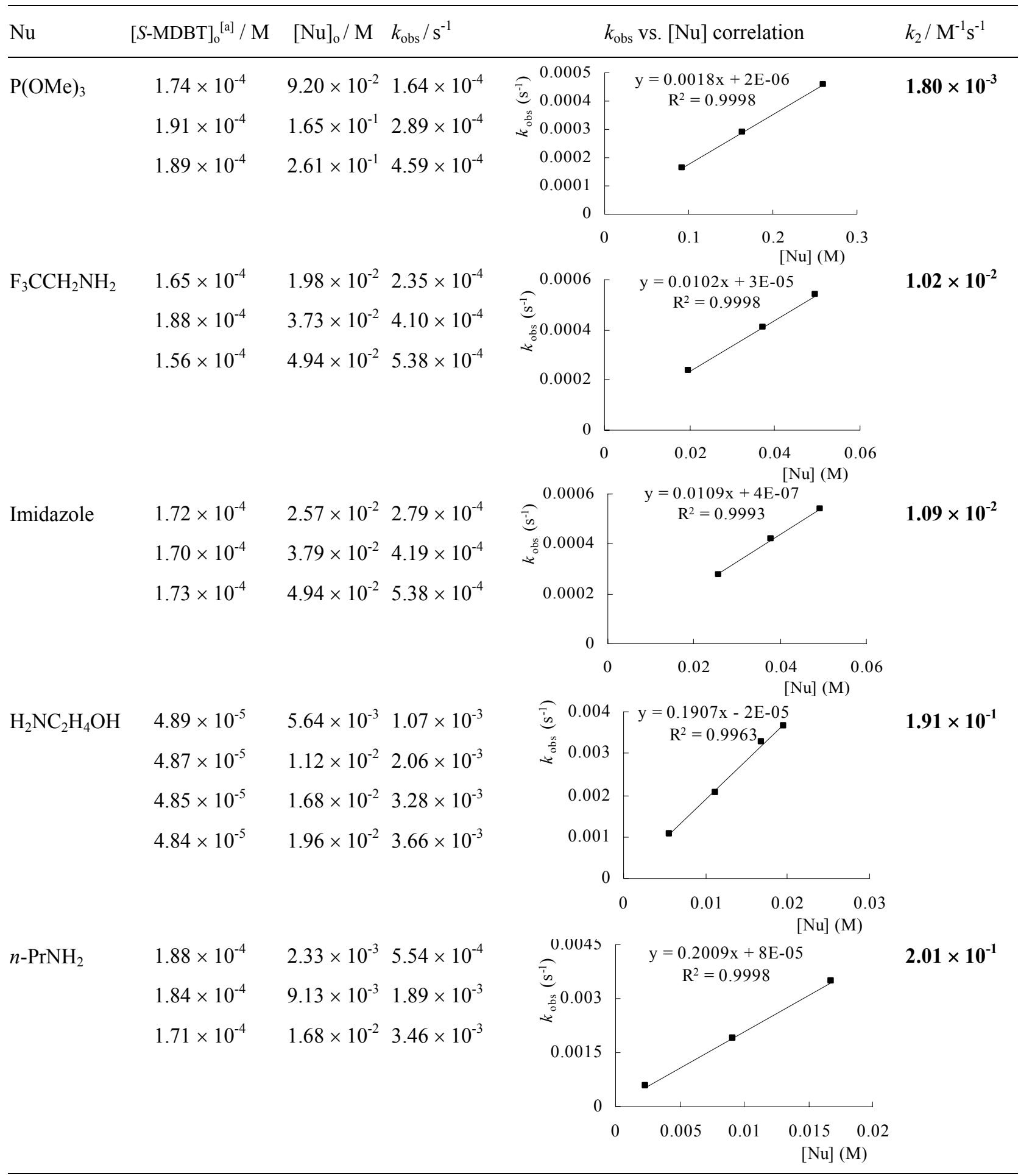




\section{Continued}

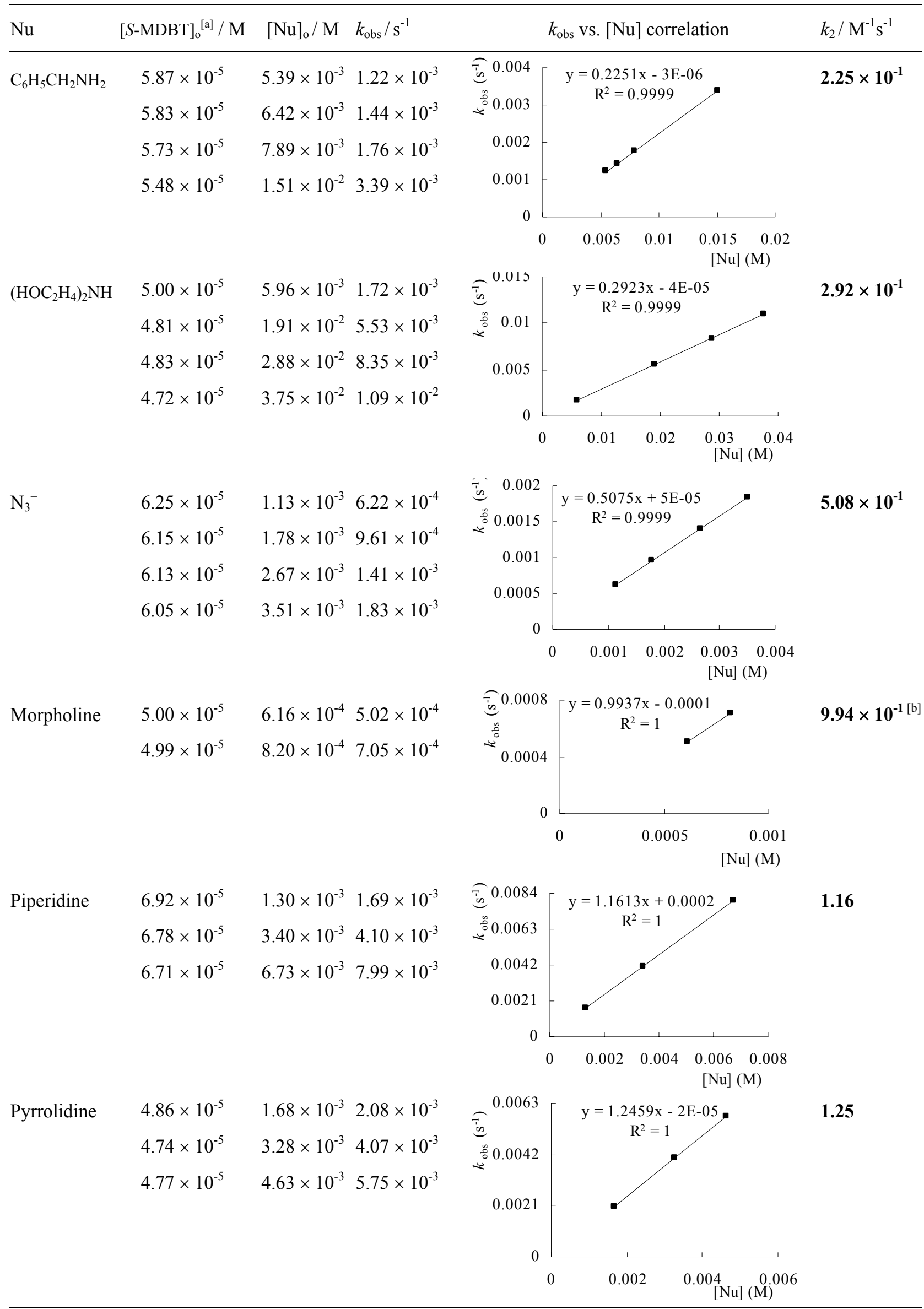




\section{Continued}

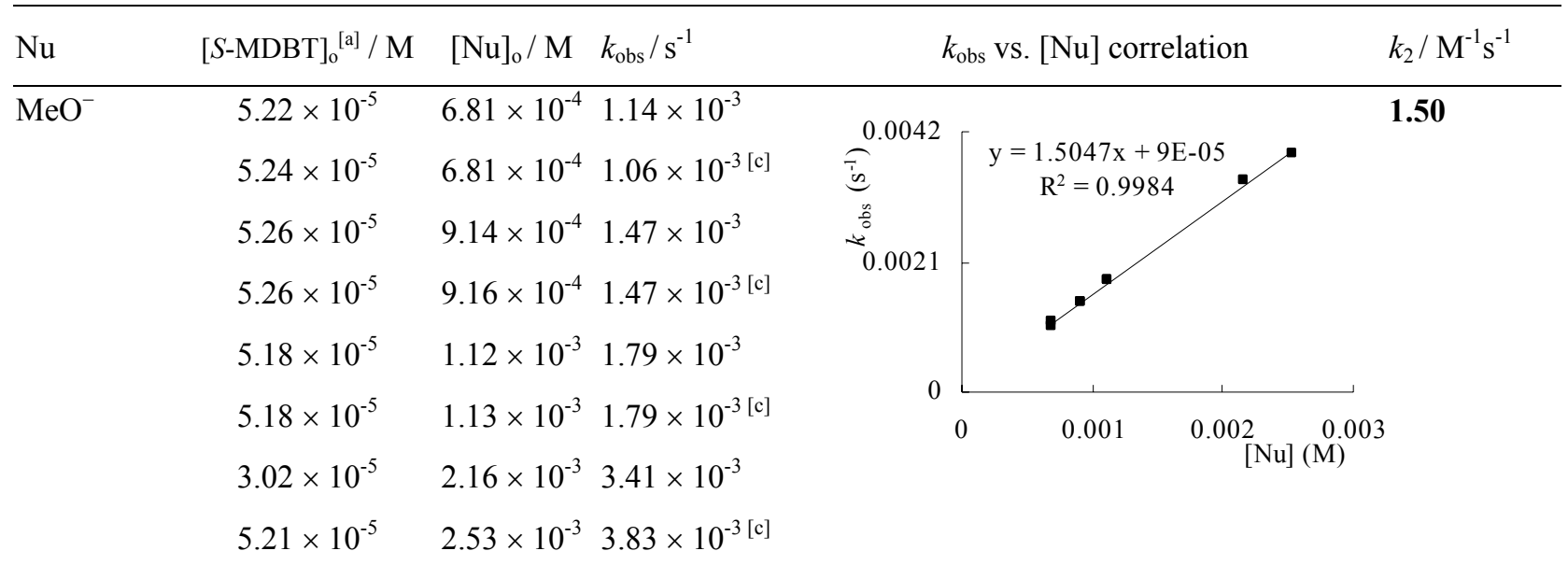

[a] $S$-MDBT stands for $S$-methyldibenzothiophenium ion; [b] The rate constant based on only two experimental points.

[c] In pure methanol.

\subsection{Kinetics of the reactions with hydroxylamine and hydrazine}

$\left(20^{\circ} \mathrm{C}\right.$, in methanol, J\&M spectrometer, evaluated at $\left.271 \mathrm{~nm}\right)$

\section{Hydroxylamine}

\begin{tabular}{|c|c|c|c|c|c|c|c|c|}
\hline $\mathrm{Nu}$ & $\begin{array}{c}{[S-\mathrm{MDBT}]_{\mathrm{o}}{ }^{[\mathrm{a}]}} \\
/ \mathrm{M}\end{array}$ & $\begin{array}{c}{\left[\mathrm{H}_{2} \mathrm{NOH} . \mathrm{HCl}\right]_{\mathrm{o}}} \\
/ \mathrm{M} \\
\end{array}$ & $\begin{array}{c}{\left[\mathrm{MeO}^{-}\right]_{\mathrm{o}}} \\
/ \mathrm{M}\end{array}$ & $\begin{array}{c}{\left[\mathrm{H}_{2} \mathrm{NOH}\right]_{\mathrm{o}}} \\
/ \mathrm{M} \\
\end{array}$ & $\begin{array}{l}k_{\mathrm{obs}} \\
/ \mathrm{s}^{-1}\end{array}$ & \multicolumn{2}{|c|}{$k_{\text {obs }}$ Vs. $[\mathrm{Nu}]^{[\mathrm{b}]}$ correlation } & $\begin{array}{c}k_{2} \\
/ \mathrm{M}^{-1} \mathrm{~s}^{-1} \\
\end{array}$ \\
\hline \multirow[t]{5}{*}{$\mathrm{H}_{2} \mathrm{NOH}$} & $6.10 \times 10^{-5}$ & $2.72 \times 10^{-3}$ & $2.70 \times 10^{-3}$ & $2.70 \times 10^{-3}$ & $3.05 \times 10^{-4}$ & \multirow[t]{2}{*}{0.0009} & \multirow{4}{*}{$\begin{array}{c}\mathrm{y}=0.1068 \mathrm{x}+3 \mathrm{E}-05 \\
\mathrm{R}^{2}=0.9978\end{array}$} & \multirow[t]{5}{*}{$1.07 \times 10^{-1}$} \\
\hline & $6.00 \times 10^{-5}$ & $4.44 \times 10^{-3}$ & $4.42 \times 10^{-3}$ & $4.42 \times 10^{-3}$ & $5.15 \times 10^{-4} \widetilde{\hat{F}_{n}}$ & & & \\
\hline & $5.89 \times 10^{-5}$ & $6.53 \times 10^{-3}$ & $6.51 \times 10^{-3}$ & $6.51 \times 10^{-3}$ & $7.18 \times 10^{-4}=$ & 0.0006 & & \\
\hline & $5.20 \times 10^{-5}$ & $7.68 \times 10^{-3}$ & $7.66 \times 10^{-3}$ & $7.66 \times 10^{-3}$ & $8.41 \times 10^{-4}$ & 0.0003 & & \\
\hline & & & & & & 0 & $0.005[\mathrm{Nu}]$ & \\
\hline
\end{tabular}

[a] $S$-MDBT stands for $S$-methyldibenzothiophenium ion; [b] $\mathrm{Nu}=\mathrm{H}_{2} \mathrm{NOH}$, hydroxylamine was freshly generated from hydroxylamine hydrochloride by neutralization with methoxide. 


\section{Hydrazine}

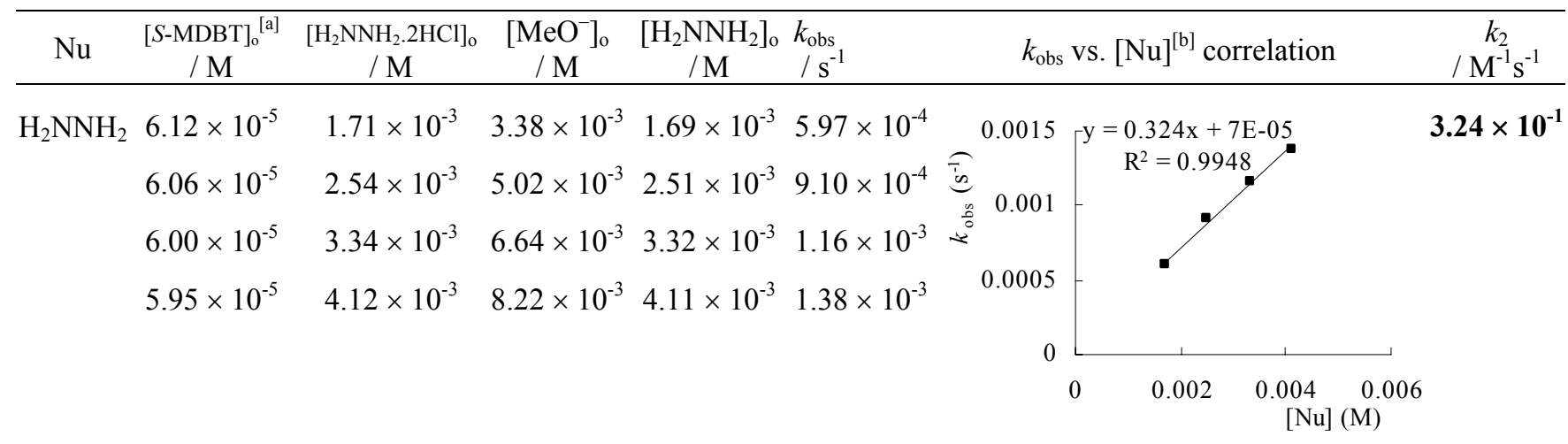

[a] $S$-MDBT stands for $S$-methyldibenzothiophenium ion; [b] $\mathrm{Nu}=\mathrm{H}_{2} \mathrm{NNH}_{2}$, hydrazine was freshly generated from hydrazine dihydrochloride by neutralization with methoxide.

\subsection{Kinetics of the reactions with carbanions}

Solutions of the carbanions were generated freshly before each kinetic measurement by mixing the $\mathrm{CH}$ acids with sodium methoxide in methanol.

The equilibrium concentrations of carbanions and methoxide were then calculated by using the $\mathrm{p} K_{\mathrm{aH}}$ or $K_{\mathrm{CH}}$ values of the $\mathrm{CH}$ acids in methanol. It has been shown previously, ${ }_{[\mathrm{S} 4 \mathrm{c}]}$ that $9 \%(\mathrm{v} / \mathrm{v})$ of the cosolvent acetonitrile do not have a noticeable effect on the $\mathrm{p} K_{\mathrm{aH}}$ values of the $\mathrm{CH}$ acids. Therefore the $K_{\mathrm{CH}}$ values [Eqs. (S2), (S3), and (S4)] which were reported in pure methanol were used to calculate the equilibrium concentrations of the carbanions and the methoxide ion.

$$
\begin{aligned}
& \mathrm{CH}+\mathrm{MeO}^{-} \stackrel{K_{\mathrm{CH}}}{=} \mathrm{C}^{-}+\mathrm{MeOH} \\
& K_{\mathrm{CH}}=K_{\mathrm{aH}} / K_{\mathrm{a}, \mathrm{MeOH}} \\
& K_{\mathrm{CH}}=\left(\left[\mathrm{C}^{-}\right] /\left([\mathrm{CH}]\left[\mathrm{MeO}^{-}\right]\right)\right.
\end{aligned}
$$

When the initial concentrations of the $\mathrm{CH}$ acids and methoxide are $[\mathrm{CH}]_{\mathrm{o}}$ and $\left[\mathrm{MeO}^{-}\right]_{\mathrm{o}}$, respectively, the equilibrium concentrations are:

$[\mathrm{CH}]=[\mathrm{CH}]_{\mathrm{o}}-\left[\mathrm{C}^{-}\right]$and $\left[\mathrm{MeO}^{-}\right]=\left[\mathrm{MeO}^{-}\right]_{\mathrm{o}}-\left[\mathrm{C}^{-}\right]$

With the known initial concentrations of the $\mathrm{CH}$ acids and methoxide and equations (S3), (S4) and (S5), it wass possible to calculate $\left[\mathrm{C}^{-}\right]$and $\left[\mathrm{MeO}^{-}\right]$.

Because of superimposed absorbances of $S$-methyldibenzothiophenium ion and carbanions, the rates of the reactions were followed at $325 \mathrm{~nm}$, which reflects the rate of the formation of dibenzothiophene. The firstorder rate constants $k_{\mathrm{obs}}\left(\mathrm{s}^{-1}\right)$ were obtained by least-squares fitting of the absorbance $A$ at $325 \mathrm{~nm}$ to the single-exponential $A_{t}=A_{0} \exp \left(-k_{\mathrm{obs}} t\right)+C$.

In general, the competing reactions of methoxide and methanol with the $S$-methyldibenzothiophenium ion have to be considered [Eq. (S6)].

$k_{\mathrm{obs}}=k_{2, \mathrm{C}}\left[\mathrm{C}^{-}\right]+k_{2, \mathrm{MeO}}\left[\mathrm{MeO}^{-}\right]+k_{1, \mathrm{MeOH}}$ 
The rate constant $k_{2, \mathrm{MeO}}$ for the reactions of methoxide with the $S$-methyldibenzothiophenium ion was determined in Section 3.1 to be $1.50 \mathrm{M}^{-1} \mathrm{~s}^{-1}$. Hence, Equation (S7) was used to subtract the contribution of the competing methoxide reaction from the observed first-order rate constants $k_{\mathrm{obs}}$.

$k_{1 \Psi}=k_{\mathrm{obs}}-k_{2, \mathrm{MeO}}\left[\mathrm{MeO}^{-}\right]=k_{2, \mathrm{C}}\left[\mathrm{C}^{-}\right]+k_{1, \mathrm{MeOH}}$

The first order rate constants $k_{1 \Psi}\left(\mathrm{s}^{-1}\right)$ from Equation (S7) were then plotted against the concentrations of the carbanions giving rise to linear correlations with the slopes corresponding to the second-order rate constants of the reactions of carbanions with the $S$-methyldibenzothiophenium ion, $k_{2, \mathrm{C}}\left(\mathrm{M}^{-1} \mathrm{~s}^{-1}\right)$.

This method of evaluating the second-order rate constants is analogous to that previously described in references [S4c, S5].

The experiments were performed with a stopped-flow instrument by mixing solutions of the carbanions in methanol with solutions of the $S$-methyldibenzothiophenium ion in acetonitrile in a $10 / 1$ mixing ratio. Because of the 10/1 mixing ratio, the effective concentrations of carbanions $\left[\mathrm{C}^{-}\right]_{\mathrm{eff}}$ and methoxide $\left[\mathrm{MeO}^{-}\right]_{\mathrm{eff}}$ were calculated from the concentrations before mixing the solutions of the nucleophiles with the solutions of the reference electrophiles by division by 1.1 .

In all the experiments, an increase of the absorbance at $325 \mathrm{~nm}$ during the first $10 \mathrm{~s}$ was observed, which was independent of the nucleophile concentrations. The same increase was observed when the solution of $S$ methyldibenzothiophenium ion in acetonitrile was mixed with pure methanol (without added $\mathrm{CH}$ acid or methoxide). This initial process was followed by a further exponential increase of the absorbance. From this part of the curve $(t>10 \mathrm{~s})$, the evaluated $k_{\text {obs }}$ showed linear dependence on the nucleophile concentrations. Therefore, only this part $(t>10 \mathrm{~s})$ was used for the evaluation of the rate constants of the reactions.

Reaction with the malononitrile ion: $k_{2, \mathrm{C}}=11.34 \mathrm{M}^{-1} \mathrm{~s}^{-1}$

$\left(20{ }^{\circ} \mathrm{C}\right.$, in $91 / 9(\mathrm{v} / \mathrm{v})$ methanol/acetonitrile, stopped-flow spectrometer, evaluated at $\left.325 \mathrm{~nm}\right)$

\begin{tabular}{|c|c|c|c|c|c|c|c|c|c|c|}
\hline \multicolumn{6}{|c|}{ Concentrations $^{[\mathrm{a}]}(\mathrm{M})$} & Rates & \multirow{3}{*}{ 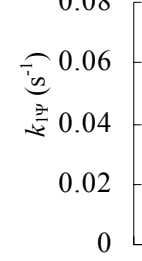 } & \multicolumn{2}{|c|}{$\begin{array}{c}\mathrm{y}=11.341 \mathrm{x}-0.0011 \\
\mathrm{R}^{2}=0.9993\end{array}$} & \\
\hline \multicolumn{4}{|c|}{ In syringes } & \multicolumn{2}{|c|}{ In reaction cell } & \multirow{2}{*}{$k_{\mathrm{obs}}\left(\mathrm{s}^{-1}\right) \quad k_{1 \Psi}\left(\mathrm{s}^{-1}\right)$} & & & & \\
\hline$[\mathrm{CH}]_{\mathrm{o}}$ & {$\left[\mathrm{MeO}^{-}\right]_{\mathrm{o}}$} & {$\left[\mathrm{C}^{-}\right]$} & {$\left[\mathrm{MeO}^{-}\right]$} & {$\left[\mathrm{C}^{-}\right]_{\mathrm{eff}}$} & {$\left[\mathrm{MeO}^{-}\right]_{\mathrm{eff}}$} & & & & & \\
\hline $8.15 \times 10^{-2}$ & $1.48 \times 10^{-3}$ & $1.45 \times 10^{-3}$ & $3.03 \times 10^{-5}$ & $1.321 \times 10^{-3}$ & $2.75 \times 10^{-5}$ & $1.43 \times 10^{-2} \quad 1.43 \times 10^{-2}$ & tan & 0.002 & 0.004 & 0.006 \\
\hline $2.04 \times 10^{-1}$ & $3.71 \times 10^{-3}$ & $3.68 \times 10^{-3}$ & $3.06 \times 10^{-5}$ & $3.344 \times 10^{-3}$ & $2.79 \times 10^{-5}$ & $3.62 \times 10^{-2} \quad 3.62 \times 10^{-2}$ & & & & M) \\
\hline $3.26 \times 10^{-1}$ & $5.93 \times 10^{-3}$ & $5.90 \times 10^{-3}$ & $3.07 \times 10^{-5}$ & $5.367 \times 10^{-3}$ & $2.80 \times 10^{-5}$ & $6.02 \times 10^{-2} \quad 6.01 \times 10^{-2}$ & & & & \\
\hline
\end{tabular}

[a] Concentration of the $S$-methyldibenzothiophenium ion in reaction cell $=2.05 \times 10^{-5} \mathrm{M} ;\left[\mathrm{C}^{-}\right]$and $\left[\mathrm{MeO}^{-}\right]$were calculated based on $K_{\mathrm{CH}}=6.00 \times 10^{2} \mathrm{M}^{-1}$. ${ }^{\left[{ }^{6} 6\right]}$ 
Reaction with the dimethyl malonate ion: $k_{2, \mathrm{C}}=14.09 \mathrm{M}^{-1} \mathrm{~s}^{-1}$

$\left(20{ }^{\circ} \mathrm{C}\right.$, in $91 / 9(\mathrm{v} / \mathrm{v})$ methanol/acetonitrile, stopped-flow spectrometer, evaluated at $\left.325 \mathrm{~nm}\right)$

\begin{tabular}{|c|c|c|c|c|c|c|c|}
\hline \multicolumn{6}{|c|}{ Concentrations $^{[\mathrm{a}]}(\mathrm{M})$} & \multicolumn{2}{|c|}{ Rates } \\
\hline \multicolumn{4}{|c|}{ In syringes } & \multicolumn{2}{|c|}{ In reaction cell } & \multirow{2}{*}{$k_{\mathrm{obs}}\left(\mathrm{s}^{-1}\right)$} & \multirow{2}{*}{$k_{1 \Psi}\left(\mathrm{s}^{-1}\right)$} \\
\hline$[\mathrm{CH}]_{\mathrm{o}}$ & {$\left[\mathrm{MeO}^{-}\right]_{\mathrm{o}}$} & {$\left[\mathrm{C}^{-}\right]$} & {$\left[\mathrm{MeO}^{-}\right]$} & {$\left[\mathrm{C}^{-}\right]_{\mathrm{eff}}$} & {$\left[\mathrm{MeO}^{-}\right]_{\mathrm{eff}}$} & & \\
\hline $8.71 \times 10^{-1}$ & $3.37 \times 10^{-3}$ & $9.48 \times 10^{-4}$ & $2.42 \times 10^{-3}$ & $8.62 \times 10^{-4}$ & $2.20 \times 10^{-3}$ & $1.56 \times 10^{-2}$ & $1.22 \times 10^{-2}$ \\
\hline $8.71 \times 10^{-1}$ & $6.74 \times 10^{-3}$ & $1.89 \times 10^{-3}$ & $4.84 \times 10^{-3}$ & $1.72 \times 10^{-3}$ & $4.40 \times 10^{-3}$ & $3.10 \times 10^{-2}$ & $2.44 \times 10^{-2}$ \\
\hline $8.71 \times 10^{-1}$ & $1.01 \times 10^{-2}$ & $2.84 \times 10^{-3}$ & $7.27 \times 10^{-3}$ & $2.58 \times 10^{-3}$ & $6.61 \times 10^{-3}$ & $4.65 \times 10^{-2}$ & $3.65 \times 10^{-2}$ \\
\hline $8.71 \times 10^{-1}$ & $1.35 \times 10^{-2}$ & $3.78 \times 10^{-3}$ & $9.69 \times 10^{-3}$ & $3.44 \times 10^{-3}$ & $8.81 \times 10^{-3}$ & $6.18 \times 10^{-2}$ & $4.85 \times 10^{-2}$ \\
\hline $8.71 \times 10^{-1}$ & $1.68 \times 10^{-2}$ & $4.73 \times 10^{-3}$ & $1.21 \times 10^{-2}$ & $4.30 \times 10^{-3}$ & $1.10 \times 10^{-2}$ & $7.72 \times 10^{-2}$ & $6.06 \times 10^{-2}$ \\
\hline
\end{tabular}

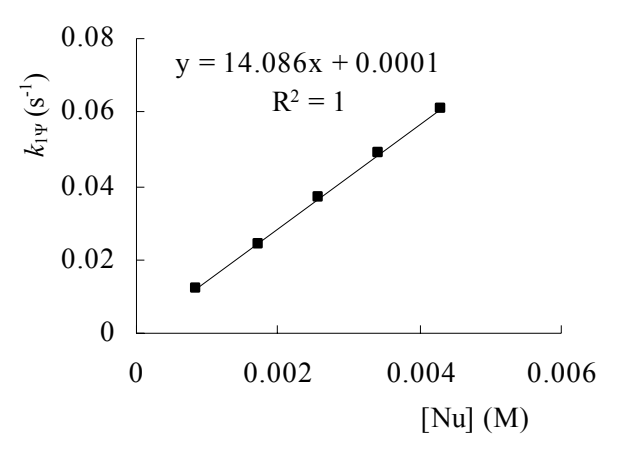

[a] Concentration of the $S$-methyldibenzothiophenium ion in reaction cell $=2.01 \times 10^{-5} \mathrm{M}$; $\left[\mathrm{C}^{-}\right]$and $\left[\mathrm{MeO}^{-}\right]$were calculated based on $K_{\mathrm{CH}}=4.50 \times 10^{-1} \mathrm{M}^{-1}{ }^{[\mathrm{S} 7]}$

\subsection{Kinetics of the reactions with propan-1-ol and propan-2-ol}

$\left(20^{\circ} \mathrm{C}, \mathrm{J} \& \mathrm{M}\right.$ spectrometer, evaluated at $\left.271 \mathrm{~nm}\right)$

The first-order rate constants $k_{1}\left(\mathrm{~s}^{-1}\right)$ of the reactions of propan-1-ol and propan-2-ol with the $S$ methyldibenzothiophenium ion were calculated as mean values of the $k_{\text {obs }}$ obtained from several runs.

\begin{tabular}{|c|c|c|c|}
\hline Solvent & {$[S-\mathrm{MDBT}]_{\mathrm{o}}^{[\mathrm{a}]} / \mathrm{M}$} & $k_{\mathrm{obs}} / \mathrm{s}^{-1}$ & $k_{1} / \mathrm{s}^{-1}$ \\
\hline \multirow[t]{3}{*}{ propan-1-ol } & $1.80 \times 10^{-4}$ & $8.50 \times 10^{-5}$ & $8.50 \times 10^{-5}$ \\
\hline & $1.81 \times 10^{-4}$ & $8.50 \times 10^{-5}$ & \\
\hline & $1.78 \times 10^{-4}$ & $8.50 \times 10^{-5}$ & \\
\hline \multirow[t]{3}{*}{ propan-2-ol } & $1.80 \times 10^{-4}$ & $6.33 \times 10^{-5}$ & $6.42 \times 10^{-5}$ \\
\hline & $1.82 \times 10^{-4}$ & $6.46 \times 10^{-5}$ & \\
\hline & $1.78 \times 10^{-4}$ & $6.47 \times 10^{-5}$ & \\
\hline
\end{tabular}

[a] $S$-MDBT stands for $S$-methyldibenzothiophenium ion 


\section{References}

[S1] H. Mayr, T. Bug, M. F. Gotta, N. Hering, B. Irrgang, B. Janker, B. Kempf, R. Loos, A.R. Ofial, G. Remennikov, H. Schimmel, J. Am. Chem. Soc. 2001, 123, 9500-9512.

[S2] D. N. Kevill, S. W. Anderson, J. Org. Chem. 1991, 56, 1845-1850.

[S3] a) S. Minegishi, H. Mayr, J. Am. Chem. Soc. 2003, 125, 286-295; b) A. D. Dilman, S. L. Ioffe, H. Mayr, J. Org. Chem. 2001, 66, 3196-3200; c) H. Mayr, R. Schneider, C. Schade, J. Bartl, R. Bederke, J. Am. Chem. Soc. 1990, 112, 4446-4454; d) H. Mayr, A. R. Ofial, Einsichten-Forschung an der LMU München 2001, 20(2), 30-33.

[S4] a) S. Minegishi, H. Mayr, J. Am. Chem. Soc. 2003, 125, 286-295; b) T. B. Phan, H. Mayr, Can. J. Chem. 2005, 83, 1554-1560; c) T. B. Phan, H. Mayr, Eur. J. Org. Chem. in print.

[S5] a) T. Bug, T. Lemek, H. Mayr, J. Org. Chem. 2004, 69, 7565-7576; b) T. Bug, H. Mayr, J. Am. Chem. Soc. 2003, 125, 12980-12986.

[S6] M. R. Crampton, T. P. Kee, J. R. Wilcock, Can. J. Chem. 1986, 1714-1720.

[S7] M. R. Crampton, J. A. Stevens, J. Chem. Soc. Perkin. Trans. 2 1991, 1715-1720. 